Helio G. Rocha Neto ${ }^{1,2}$

(1) https://orcid. org/0000-0002-7342-0634

Cátia Maria Mathias ${ }^{1}$

Ohttps://orid.org/0000-0002-8321-1393

Antonio Egidio Nardi

(- https://orcid.org/0000-0002-2152-4669

Marleide Mota Gomes ${ }^{3}$

(- https://orcid.org/0000-0001-8889-2573

Maria Tavares Cavalcanti

Ohttps://orcid.org/0000-0003-1872-4210

\section{The Weekly Study Meeting in Psychiatry of the Institute of Psychiatry - Federal University of Rio de Janeiro: 70-years of culture and scientific dissemination}

\author{
O Encontro Semanal do Centro de Estudos do Instituto de Psiquiatria da \\ Universidade Federal do Rio de Janeiro: 70 anos de divulgação cultural e científica
}

DO1: $10.1590 / 0047-2085000000357$

\begin{abstract}
Objective: To describe the history of creation, development, and topics covered by the Study Center of the Institute of Psychiatry of the Federal University of Rio de Janeiro (CE - IPUB/UFRJ) over its 70 years. Methods: Research in newspapers of the Hemeroteca Brasileira Digital, internal documents of IPUB/UFRJ, and interviews with eyewitnesses of the functioning of the CE. Results: The Study Center has been operating on an uninterrupted basis for 70 years, every week. 472 events have been identified since the founding of the CE, but numerous other meetings have taken place. The findings were described in three major groups: 1 . Academic meetings in the first half of the 20th century and insertion of the CE in the history of IPUB; 2. Topics discussed and presentations; 3. Changes in periodicity and format. Conclusions: The CE produces cultural and scientific dissemination continuously since its foundation. The type and format of events have changed over time, adapting to the needs of their community, but always serving as an important beacon for the training of specialists in mental health, dissemination of research, and tendencies about psychiatry worldwide, Latin America, and Brazil.
\end{abstract}

\section{KEYWORDS}

Scientific communication and diffusion, history of medicine, psychiatric hospitals, scientific and educational events, lecture.

\section{RESUMO}

Objetivo: Descrever a história da criação e do desenvolvimento e os temas abordados pelo Centro de Estudos do Instituto de Psiquiatria da Universidade Federal do Rio de Janeiro (CE - IPUB/UFRJ) ao longo de seus 70 anos. Métodos: Pesquisa em jornais da Hemeroteca Brasileira Digital, documentos internos do IPUB/UFRJ e entrevistas com testemunhas oculares do funcionamento do CE. Resultados: $O$ Centro de Estudos funciona de maneira ininterrupta há 70 anos, semanalmente. Desde sua fundação, 472 eventos foram identificados, mas outros inúmeros encontros ocorreram. Os achados foram divididos e descritos em três grandes grupos: 1. Encontros acadêmicos na primeira metade do século XX e inserção do CE na história do IPUB; 2. Temas debatidos e apresentações; 3. Mudanças de periodicidade e formato. Conclusões: O CE produz divulgação cultural e científica continuamente desde sua fundação. O tipo e o formato dos eventos mudaram ao longo do tempo, adaptando-se às necessidades de sua comunidade, mas sempre servindo como um importante farol para a formação de especialistas em saúde mental e divulgação de pesquisas, apresentando as tendências da psiquiatria mundial, latino-americana e brasileira.

\section{PALAVRAS-CHAVE}

Comunicação e divulgação científica, história da medicina, hospitais psiquiátricos, eventos científicos e de divulgação, aula.
Received in: Mar/20/2021. Approved in: Aug/10/2021

1 Federal University of Rio de Janeiro, Institute of Psychiatry, Rio de Janeiro, RJ, Brazil.

2 Lisbon University, Lisbon Medicine Faculty, Lisbon, Portugal.

3 Federal University of Rio de Janeiro, School of Medicine, Institute of Neurology Deolindo Couto, Epilepsy Program, Rio de Janeiro, RJ, Brazil.

Address for correspondence: Helio Rocha Neto. Rua Dr. Armando Salles de Oliveira, 150, Boqueirão - 11050-071 - Santos, SP, Brasil. E-mail: hgrochaneto@gmail.com 


\section{INTRODUCTION}

Making scientific data accessible to public is a challenge in academic research. Nowadays, the scientific world could use internet to publish articles, podcasts, online classes, streaming videos, and so on. However, internet is a recent technology, so academics have previously used other ways to publicize their findings. One of them, as old as the Ancient Greeks, are public lectures during academic meetings.

In Rio de Janeiro, it is usual for college and postgraduation courses to have a recurrent meeting where scientific production can be shared with partners, but its exact origins are not clear. Despite that, it is possible that the "Centro de Estudos" (CE - study center, in a literal translation, but better translated as Weekly Meeting of the Institute) of Rio de Janeiro's Federal University Institute of Psychiatry (Instituto de Psiquiatria da Universidade Federal do Rio de Janeiro - IPUB/UFRJ) to be the oldest regular public academic meeting about mental health still in activity in Brazil.

In 2021, IPUB's CE complete 70 years from its first regular week-meeting, on February, 26th of 1951'. To celebrate this historical mark, it is here presented a brief report to elucidate its origins, meeting themes, and development as it has had an enormous impact on Brazilian and international psychiatry.

\section{METHODS}

"Hemeroteca Digital Brasileira" (HDB), a Brazilian repository of documents from the last 2 and a half centuries, was screened for the CE events. Also, CE registries in IPUB files were accessed, as Jornal Brasileiro de Psiquiatria (JBP) previous issues, and an interview with one of the actual coordinators (Andrea Deslandes). Two CE coordinators of the last 30 years were also interviewed, looking for information about the format that was not available in the documents. Three authors are direct eyewitnesses of the recent CE history as former director (MTC), researcher (AEN), and student (HRN), and provided memory reports.

\section{RESULTS}

The HDB retrieved 76 unique CE events from February 26th of 1951 to July 7th of 1987 . Most events identified were during the '50s and '60s. An intern document reported all the 46 CE events during the year 1963, and further search in the physical file of JBP during the 60's identified other 19 events. Finally, CE physical reports were found, starting in 75 to 2001, adding 250 entries to our sample, and electronic repositories contributed with other 91 entries from 2017 to 2020. This results may be observed in figure 1 .

The total amount of unique entries was 472 since some entries were represented in the HDB, inner IPUB documents, and in JBP publications. Our results are described in three main themes: 1. Academic meetings in the first half of the 20th century and CE Insertion in IPUB's history; 2. Debated themes and presentations; 3. Periodicity and format changes. A detailed description of each event is out of the scope of the present report, but the resulting list may be requested by the direct authors' contact.

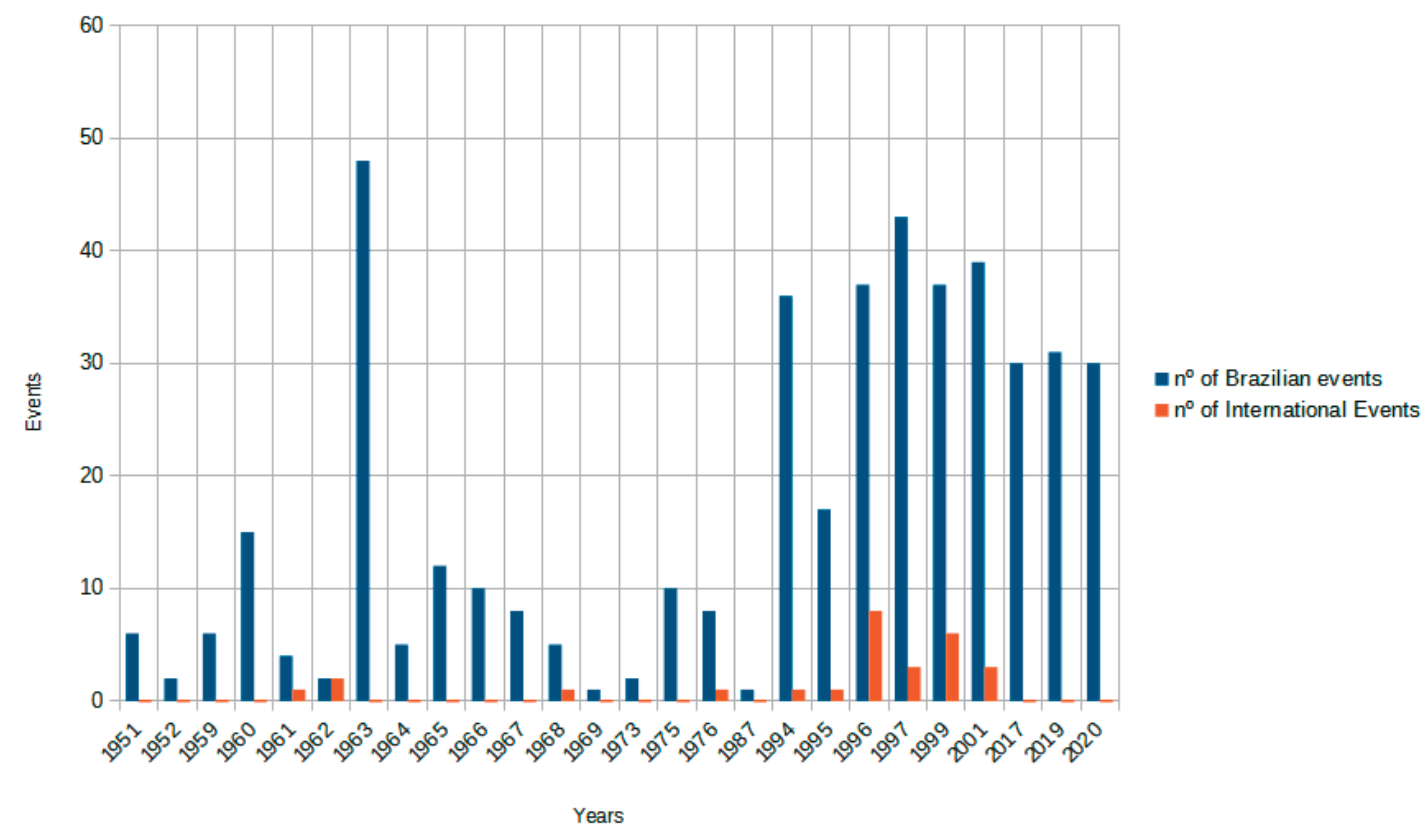

Figure 1. Study Center of Events obtained from 1951 to 2020. 


\section{DISCUSSION}

\section{Academic meeting in the first half of the 20th century and CE Insertion in IPUB's history}

HDB has entries about scientific meetings being held in Brazil since 1906, so, our findings suggests that academic sessions were held regularly by medical societies, at least since the very beginning of the $20^{\text {th }}$ century. We also find entries in HDB about scientific sessions held in other countries and Brazilian states in the first half of the $20^{\text {th }}$ century. It was in 1906 where was reported by the journal "Correio da Manhã" a call for a "study center" in social sciences to be held on September 23rd², among others similar events. In the next year, the Brazilian Society of Neurology, Psychiatry and Legal Medicine (BSNPLM) would be founded, and a report from the "Jornal do Comércio" on November 16th of 1917 confirms that a regular scientific meeting was held at that institution ${ }^{3}$.

These sessions were carried at "Hospital Nacional de Alienados" ${ }^{\prime \prime}$, in which the observation pavilion would be later transformed in IPUB'. It was not possible to identify, however, if such meetings have any influence on the further foundation of CE in 1951. Also, it is necessary to say that "Centro de Estudos" was not the usual name of such meetings in Brazil at the beginning of the 20th century, being used for any educational meetings. Between the found entries in HDB using "Centro de Estudos" as the search string, it is possible to find references to universities, schools, and recurrent Kardecist spiritual sessions.

"Hospital Nacional de Alienados" observation pavilion became IPUB as part of the concession of the entire facility to "Universidade do Brasil", the previous name o UFRJ, in $1938^{4}$. No entries about academic meetings were found during its first 12 years, from 1938 to 50. Then in February 26th of 1951, it was funded the CE of IPUB, with Maurício de Medeiros as its president, and Alfredo de Moraes Coutinho Filho, Jurandir Manfredini and William Asmar as permanent members?.

The inauguration of CE is probably part of the changes implemented by Maurício de Medeiros to improve IPUB's assistance and academic practices. During his period as IPUB director, he structured the offered courses, organized the insertion of psychiatry rotation in the medicine course, and developed a robust curriculum for graduation and specialization courses. In this scenario, the creation of an instrument for public discussion and scientific dissemination seems mandatory ${ }^{5}$.

Although it was not possible to find entries of all the sessions held since its foundation, CE has been occurring with regularity since 1951. During these 70-years, it was a scientific beacon for all academic purposes in IPUB, where the most relevant researches and communications were held and publicized for the university community.

\section{Debated themes and presentations}

During the entire period, four main types of meetings were found: "Thematic presentation", "Bibliographic Reviews", "Homage sessions" and "Clinical case". The first was the most prevalent, with 316 entries, followed by 39 "Clinical case", 29 "Homage sessions", and 25 "bibliographic reviews". "Thematic presentation" represents speeches about specific themes or research by invited professionals or by researchers from $I P U B$, UFRJ, or other academic instances. It still is the main type of CE presentation until nowadays $s^{6}$. A clear prevalence of a thematic subject, in a specific time span, could not be identified in the list of the events, that could refer to a scientific shift in psychiatry. All decades have psychoanalysis/ psychodynamic, biological and social scientific background presentations. The full list can be obtained in CE site, as a result of the present research.

Bibliographic reviews were a brief abstract of articles published in scientific journals selected by the organizers and may have represented the only access for regular students to such bibliography back in the '50s and '60s. Clinical case presentations were held by the students, but eventually by the main organizers themselves, usually with cases being followed in the inpatient settings. Late in the sixties, it is possible to observe a pattern so the case to be presented were sequentially presented by inpatient (male and female) and outpatient unity and its coordinators.

In the inner document from CE 1963 sessions $^{7}$, it is said that was expected that 2 sessions to be reserved for clinical cases, 1 for thematic presentation, and 1 for bibliographic reviews. Bibliographic reviews and clinical cases are no longer part of IPUB CE's, but the exact time when it stopped was not found. In our interviews, an eyewitness said that in 1986 they were no longer part of the recurrent meetings ${ }^{8}$. In our documentation, both events are part of CE only in the '50s and '60s, but it does not mean that the activity was abandoned.

In a recent Ph.D. thesis, an eyewitness reported that Prof Leme Lopes waged a recurrent clinical session every week, long before 1986. Also, in 1994, during João Ferreira's tenure, clinical cases became recurrently exposed in an especial weekly meeting on Tuesdays, in the format that is still being held nowadays (MTC).

Homage sessions are a heterogeneous group, composed basically by commemorative sessions about institutional anniversaries, the memory of an important retired or deceased professor, and inauguration or celebration of an academic year's start or ending. Sometimes those sessions were held together with a thematic presentation, but in the early years of CE seems to have been usual speeches from doctors that were students or friend of the honored.

CE was also the stage for some very relevant presentations and historical developments in Brazilian psychiatry. It was in CE 
that the idea of a national entity for psychiatry was conceived, resulting in the posterior foundation of the Brazilian Association of Psychiatry. It has been also a hub for the dissemination of international research about mental health in Brazil.

The first international speaker may have been G. Davi, from the neuropsychiatric research group of the medical research council in London, England. A note from "Diário de Notícias" on May 5th of 1961 invited the community for a lecture about "some issues in experimental psychiatry"10. Lectures from international researchers or Brazilian doctors studying in foreign universities were relatively common, and nineteen entries were identified as foreign speechers in our research. Some of these lectures were administered in English ${ }^{10}$, but probably also in Spanish and French. Among important speeches, we may cite Hagoup Akiskal, Thomas Ban, and Bruno Cerleti, among many other world-known psychiatric mentors (AEN).

Other entries are harder to categorize, but worth mention. It was obtained data from meetings aimed to describe congresses, symposiums, and foreign services visiting, usually held by the staff who attended it. There were 44 events about intern congress, symposiums, and forums held with CE as a basis, and at least since 1996 Ph.D. thesis and Master of Science essays were part of CE regular schedules ${ }^{8}$. Another eyewitness remembered that until 2014 these post-graduation events were recurrent (HRN), but it was not identified after 2017 when CE become restricted to Thematic presentation and few Homage sessions ${ }^{8}$.

\section{Periodicity and format changes}

The first session of CE was held at the Institute amphitheater, today called "Henrique Roxo Amphitheater". Back then, the sessions were held monthly, but in 1961 we found entries every two weeks, and in 1963 it became weekly. Today, and at least since 1982, CE is based in "Leme Lopes" auditorium.

It is not clear if there has ever been any access restriction, but journal notes seemed to be directed for physicians in the 50s. In the 1963 document it is explicitly stated that other health professionals as nurses, psychologists, and social workers were welcome for the sessions ${ }^{7}$, and in 1964 such invitations were found in journal notes ${ }^{11}$. It could be argued if $C E$ is a scientific dissemination event since its language is certainly not aimed at the lay public, but it seems to have been very accessible for all at least since 1963.

Since 2019, CE became integrated with the extension course "Connected Education in Mental Health and Psychiatry" (EPSAMP) and is part of the post-graduation courses in UFRJ'. It also integrates the monthly events of the Brazilian University Web of Telemedicine, both as a content provider and consumer ${ }^{12}$. In the year 2020, with the COVID-19 outbreak, CE starts to be transmitted by EPSAMP YouTube channel by streaming, with a mean audience of 200 but reaching more than 500 in some events ${ }^{6,13}$.

\section{CONCLUSION}

In the last 70-years, CE developed from a local-based scientific meeting to a full, internationally web-based, free recurrent event of mental health information entity. During its meeting, some important events of Brazilian psychiatry were developed, and a relevant exchange of international experiences and research alliances was also held. In a period before the internet, the only access to field tendencies and scientific update for mental health professional were during these meetings, that were free and readily accessible for all the community.

It has also significantly contributed to the formation of many generations of mental health professionals and influenced directly the routes of Brazilian psychiatry practice, research, and organization. The Institute of Psychiatry is proud of such achievements from the Brazilian academy and celebrates its contributions for generations of mental health professionals.

\section{INDIVIDUAL CONTRIBUTIONS}

Helio Rocha Neto - Conceived the methodology, searched and summarized the findings and wrote the manuscript.

Catia Matias - Helped in the methodology concept, searched and retrieved many findings and took part in the final manuscript writing the findings summary and took part in the final manuscript writing.

Antonio Egidio Nardi and Marleide Mota Gomes - Conceived the thematic concept and oriented draft elaboration.

Maria Tavares Cavalcanti - Obtained interview information, gathered interpersonal data and oriented the findings summary and took part in the final manuscript writing.

\section{CONFLICT OF INTEREST}

Helio Rocha Neto receives a PhD grant form Capes.

\section{ACKNOWLEDGMENTS}

We would like to thanks Prof. Dr. Andrea Deslandes for the their valuable contributions.

\section{REFERENCES}

1. Reuniões. Centro de Estudos do Instituto de Psiquiatria da Universidade do Brasil. Correio da Manhã. 1951 Feb 27. Available from: http://bndigital.bn.br/hemeroteca-digital/ 
2. Operária V. Centro de Estudos sociais. Correio da Manhã. 1936 Sep 23. Available from: http://bndigital.bn.br/hemeroteca-digital/

3. Sociedade Brasileira de Neurologia P e ML. 100 aniversário. Jornal do Commercio. 1917 Nov 16. Available from: http://bndigital.bn.br/hemeroteca-digital/

4. Venâncio ATA. Psychiatric science and social service policy: the creation of the University of Brazil's Institute of Psychiatry. Hist Cienc Saude Manguinhos. 2003;10(3):883-900.

5. Mathias CM. O Pavilhão de Observação na psiquiatria do Distrito Federal: a gestão de Henrique Roxo (1921-1945) [dissertação]. Rio de Janeiro: Fundação Oswaldo Cruz, Casa de Oswaldo Cruz; 2017.

6. Deslandes A. Personal communication. 2021.

7. Lopes JL. Relatório Ano 1963. Rio de Janeiro; 1963.

8. Cavalcanti MT. Personal communication. 2021
9. Bezz SR. A apresentação de pacientes na transmissão da psicanalise e a formação profissional em saúde mental [tese]. Rio de Janeiro: Universidade Federal do Rio de Janeiro; 2021.

10. Centro de Estudos do Instituto de Psiquiatria da UB. Diário de Notícias. 1961 May 5. Available from: http://bndigital.bn.br/hemeroteca-digital/

11. JB A. Reuniões. Jornal do Brasil. 1964 May 13. Available from: http://bndigital.bn.br/ hemeroteca-digital/

12. Rede Universitária de Telemedicina [Internet]. [cited 2021 Feb 22]. Available from: https:// rute.rnp.br/

13. EPSAMP - IPUB/UFRJ - YouTube [Internet]. [cited 2021 Feb 22]. Available from: https:// www.youtube.com/channel/UCmbzpkpVj_I2YSM51ulZZ9A

\section{Supplement}

The Study Center of the Institute of Psychiatry of the Federal University of Rio de Janeiro events from 1951 to 2020

\begin{tabular}{|c|c|c|c|c|c|}
\hline Date & Event type & Presented by & Theme & Journal/Font & Event place \\
\hline $26 / 02 / 51$ & Homage & & & Correio da Manhã; Diário Carioca & IPUB's head office \\
\hline $21 / 03 / 51$ & $\begin{array}{l}\text { Thematic } \\
\text { presentation }\end{array}$ & $\begin{array}{l}\text { Dr Adriano Cruz Ferreira; } \\
\text { Dr Humberto Alexandre }\end{array}$ & $\begin{array}{l}\text { Endocrinology and Psychiatry; Epileptic } \\
\text { petit mal seizures and situation neurosis }\end{array}$ & $\begin{array}{l}\text { Jornal do Comércio; Diário de Notícias; } \\
\text { Tribuna da imprensa; Diário Carioca }\end{array}$ & IPUB's Amphitheater \\
\hline $13 / 04 / 51$ & $\begin{array}{l}\text { Thematic } \\
\text { presentation }\end{array}$ & $\begin{array}{l}\text { Dr Alfredo Morais Coutinho; } \\
\text { Dr Almir Almeida Guimarães }\end{array}$ & $\begin{array}{l}\text { Considerations about dipsomania: a } \\
\text { case; About reactive tests in } \\
\text { electroencephalography }\end{array}$ & Correio da Manhã; Jornal do Comércio & IPUB's Amphitheater \\
\hline 05/05/51 & Homage & $\begin{array}{l}\text { Profs Drs Mauricio } \\
\text { Medeiros, Moraes Coutinho, } \\
\text { Jurandir Manfredini, Iracy } \\
\text { Doyle, Henrique de Novaes, } \\
\text { Henrique Roxo }\end{array}$ & In memory of Prof Flavio de Souza & 0 Jornal; Diário de notícias & $\begin{array}{l}\text { Brazilian's Press } \\
\text { Association } \\
\text { Auditorium }\end{array}$ \\
\hline $28 / 05 / 51$ & $\begin{array}{l}\text { Thematic } \\
\text { presentation }\end{array}$ & $\begin{array}{l}\text { Dr Adriano Cruz Ferreira, } \\
\text { Dr Alfredo Morais Coutinho } \\
\text { and interno Lourival F } \\
\text { Coimbra }\end{array}$ & $\begin{array}{l}\text { Clinical and anatomopathological } \\
\text { aspects of a case of coma in a } \\
\text { hysterical patient }\end{array}$ & $\begin{array}{l}\text { Tribuna da imprensa; Jornal do } \\
\text { Comércio; Correio da manhã }\end{array}$ & IPUB's Amphitheater \\
\hline $26 / 10 / 51$ & $\begin{array}{l}\text { Thematic } \\
\text { presentation }\end{array}$ & Dr Samuel Freitas & Simulation in a hysterical patient & Jornal do Brasil; Jornal do comércio & $\mathrm{N} / \mathrm{A}$ \\
\hline $26 / 05 / 52$ & Clinical case & $\begin{array}{l}\text { Dr Nobre de Melo and } \\
\text { Fernando Nogueira de } \\
\text { Souza }\end{array}$ & Clinical case discussion & Jornal do Comércio & N/A \\
\hline $31 / 08 / 52$ & Clinical case & N/A & $\begin{array}{l}\text { A case of cerebral neoplasia; Use of } \\
\text { Tulserol to treat Neurosis }\end{array}$ & Jornal do Comércio; A noite & IPUB's Amphitheater \\
\hline 09/01/59 & $\begin{array}{l}\text { Thematic } \\
\text { presentation }\end{array}$ & $\begin{array}{l}\text { Dr Eustachio Portella Nunes } \\
\text { Filho }\end{array}$ & Freud and Heidegger & Correio da Manhã & IPUB's head office \\
\hline $15 / 05 / 59$ & $\begin{array}{l}\text { Thematic } \\
\text { presentation }\end{array}$ & $\begin{array}{l}\text { Dr Walderedo Ismael de } \\
\text { Oliveira and Ernesto La } \\
\text { Porta }\end{array}$ & $\begin{array}{l}\text { Psychotic groups and the psychotic } \\
\text { contents of the group situation }\end{array}$ & Correio da Manhã & $\mathrm{N} / \mathrm{A}$ \\
\hline $19 / 06 / 59$ & $\begin{array}{l}\text { Thematic } \\
\text { presentation }\end{array}$ & $\begin{array}{l}\text { Dr Manoel Novaes, Dr } \\
\text { Carvalhal and Dr Newton } \\
\text { Prates Lima; Prof Dr José } \\
\text { Leme Lopes }\end{array}$ & $\begin{array}{l}\text { Diagnostic considerations about a case } \\
\text { of hemilateral motor disorders; Some } \\
\text { considerations about the psychological } \\
\text { medical problem of death }\end{array}$ & Correio da Manhã & $\mathrm{N} / \mathrm{A}$ \\
\hline $10 / 07 / 59$ & $\begin{array}{l}\text { Thematic } \\
\text { presentation }\end{array}$ & $\begin{array}{l}\text { Dr Arlindo Ribeiro Fraga; Dr. } \\
\text { J. P. Carvalhal }\end{array}$ & $\begin{array}{l}\text { Attempted Psychotherapy with a } \\
\text { schizophrenic patient; Amyloidosis of } \\
\text { the Nervous System }\end{array}$ & Correio da Manhã; Jornal do Comércio & $\mathrm{N} / \mathrm{A}$ \\
\hline $14 / 08 / 59$ & $\begin{array}{l}\text { Thematic } \\
\text { presentation }\end{array}$ & Prof. Paulo Lacaz & Some aspects of cerebral biochemistry & Diário de notícias; Correio da Manhã & $\mathrm{N} / \mathrm{A}$ \\
\hline $11 / 09 / 59$ & $\begin{array}{l}\text { Thematic } \\
\text { presentation }\end{array}$ & $\begin{array}{l}\text { Dr Paulo Carvalhal; Dr Luis } \\
\text { Cerqueira }\end{array}$ & $\begin{array}{l}\text { Brain biopsy in a case of dementia; } \\
\text { Artistic production of a schizophrenic }\end{array}$ & Correio da Manhã; Diário de Notícias & $\mathrm{N} / \mathrm{A}$ \\
\hline $10 / 02 / 60$ & $\begin{array}{l}\text { Thematic } \\
\text { presentation }\end{array}$ & Iza Alpoim da Matta & $\begin{array}{l}\text { Neurotic and environmental factors in } \\
\text { depressions }\end{array}$ & & \\
\hline
\end{tabular}




\begin{tabular}{|c|c|c|c|c|c|}
\hline Date & Event type & Presented by & Theme & Journal/Font & Event place \\
\hline $16 / 02 / 60$ & $\begin{array}{l}\text { Thematic } \\
\text { presentation }\end{array}$ & $\begin{array}{l}\text { Ernesto La Porta; Wilson } \\
\text { Chebabi and Francisco } \\
\text { Pacheco }\end{array}$ & $\begin{array}{l}\text { Landmarks of reference in group } \\
\text { psychotherapy. Evolution of a group of } \\
\text { psychotics; Observer situation in the } \\
\text { therapeutic group }\end{array}$ & Reuniões semanais, abertas ao público & \\
\hline 19/02/60 & $\begin{array}{l}\text { Thematic } \\
\text { presentation }\end{array}$ & Wasillu Chuc & $\begin{array}{l}\text { Delimitation and classification of } \\
\text { depressive states }\end{array}$ & & \\
\hline $11 / 03 / 60$ & $\begin{array}{l}\text { Thematic } \\
\text { presentation }\end{array}$ & José Leme Lopes & $\begin{array}{l}\text { Environmental and neurotic factors of } \\
\text { depressive states }\end{array}$ & & \\
\hline 25/03/60 & $\begin{array}{l}\text { Thematic } \\
\text { presentation }\end{array}$ & José Leme Lopes & $\begin{array}{l}\text { Report about the colloquy of depressive } \\
\text { states held at Buenos Aires University }\end{array}$ & & \\
\hline 08/04/60 & $\begin{array}{l}\text { Thematic } \\
\text { presentation }\end{array}$ & Paulo Carvalhal & Vegetative Syndrom & & \\
\hline $13 / 05 / 60$ & $\begin{array}{l}\text { Thematic } \\
\text { presentation }\end{array}$ & Horus Vital Brasil & Sulivan's contibute to psychoanalysis & & \\
\hline 22/07/60 & $\begin{array}{l}\text { Thematic } \\
\text { presentation }\end{array}$ & José Leme Lopes & $\begin{array}{l}\text { Impression of a visit to European } \\
\text { psychiatric clinics }\end{array}$ & & \\
\hline $10 / 08 / 60$ & $\begin{array}{l}\text { Thematic } \\
\text { presentation }\end{array}$ & Fernando Negrão Prado & $\begin{array}{l}\text { General aspects about Psychiatry in } \\
\text { Portugal }\end{array}$ & & \\
\hline 19/08/60 & $\begin{array}{l}\text { Thematic } \\
\text { presentation }\end{array}$ & José Caruso Madalena & $\begin{array}{l}\text { Previous note on the use of librium at } \\
\text { the IPUB's clinic }\end{array}$ & & \\
\hline $16 / 09 / 60$ & $\begin{array}{l}\text { Thematic } \\
\text { presentation }\end{array}$ & $\begin{array}{l}\text { Silvia Souza Barros; José } \\
\text { Caruso Madalena; }\end{array}$ & $\begin{array}{l}\text { Impressions of a visit to the European } \\
\text { Union; About a case of librium-solved } \\
\text { schizophrenia; }\end{array}$ & & \\
\hline $14 / 10 / 60$ & $\begin{array}{l}\text { Thematic } \\
\text { presentation }\end{array}$ & $\begin{array}{l}\text { Maurício Schiller; José de } \\
\text { Castro Martins }\end{array}$ & Cholinesterase; A case study of Hysteria & & \\
\hline $18 / 11 / 60$ & $\begin{array}{l}\text { Thematic } \\
\text { presentation }\end{array}$ & $\begin{array}{l}\text { Walderedo I de Oliveira, } \\
\text { Heitor de Andrade Lima }\end{array}$ & $\begin{array}{l}\text { Integration problems of new patients in } \\
\text { the therapeutic group }\end{array}$ & & \\
\hline $30 / 11 / 60$ & Homage & Dr Ulysses Vianna Filho & $\begin{array}{l}\text { Homage to the memory of Prof Antônio } \\
\text { Austregésilo }\end{array}$ & & \\
\hline 23/12/60 & $\begin{array}{l}\text { Thematic } \\
\text { presentation }\end{array}$ & $\begin{array}{l}\text { Elvira Cortes Camacho; } \\
\text { Vera Del'Amico de Almeida }\end{array}$ & $\begin{array}{l}\text { Clinical study of ten cases of inveterate } \\
\text { alcoholism; Chlorpromazine } \\
\text { impregnation in ten IPUB patients }\end{array}$ & & \\
\hline 05/05/61 & $\begin{array}{l}\text { Thematic } \\
\text { presentation }\end{array}$ & Dr G. Davi & $\begin{array}{l}\text { Some problems of experimental } \\
\text { Psychiatry }\end{array}$ & Diário de notícias; & IPUB's Amphitheater \\
\hline $26 / 05 / 61$ & $\begin{array}{l}\text { Thematic } \\
\text { presentation }\end{array}$ & $\begin{array}{l}\text { Dr Henrique de Novais; } \\
\text { Dra Vera Deli'Amico de } \\
\text { Almeida; Dr Giadriote } \\
\text { D'Alva Parente; Dr José } \\
\text { Carlos Corpilovsky; Dr } \\
\text { Ulisses Viana Filho and Dra } \\
\text { Inocência Kramer Krank; Dr } \\
\text { Mauricio Brandon; Prof Dr } \\
\text { Bernardo Henrique de } \\
\text { Nunes Couto }\end{array}$ & $\begin{array}{l}\text { Catamnosis of a series of patients } \\
\text { impregnated with chlorpromazine; } \\
\text { Result of chlorpromazine impregnation } \\
\text { in schizophrenics; Previous note on } \\
\text { trifluoropromazine impregnation; } \\
\text { Characteristics of levomepromazine } \\
\text { impregnation; Electroencephalographic } \\
\text { aspects of levomepromazine } \\
\text { impregnation; Oxiriasis and suppressive } \\
\text { activity in the course of administration } \\
\text { of high doses of chlorpromazine and } \\
\text { levomepromazine; Research film on } \\
\text { chlorpromazine impregnation }\end{array}$ & & IPUB's Amphitheater \\
\hline 23/06/61 & $\begin{array}{l}\text { Thematic } \\
\text { presentation }\end{array}$ & $\begin{array}{l}\text { Dr Edmundo Maia; } \\
\text { Prof José Leme Lopes; } \\
\text { Dr Caruso Madalena }\end{array}$ & $\begin{array}{l}\text { Psychiatric care in Brazil; Teaching of } \\
\text { Medical Psychology and psychiatric } \\
\text { clinic to medical students; Clinical trials } \\
\text { with psychotropic drugs win9.212-A } \\
\text { and R04-04-03 }\end{array}$ & Diário de notícias; & IPUB's Amphitheater \\
\hline 09/07/61 & $\begin{array}{l}\text { Bibliography } \\
\text { review }\end{array}$ & $\begin{array}{l}\text { Prof. Leme Lopes; } \\
\text { Dr. Wilson Chebabi and } \\
\text { Dr José Carlos Carpilovsky }\end{array}$ & $\begin{array}{l}\text { Literature review, 1st Latin American } \\
\text { congress of psychiatry impressions. }\end{array}$ & Diário de notícias; & IPUB's Amphitheater \\
\hline 22/12/61 & $\begin{array}{l}\text { Thematic } \\
\text { presentation }\end{array}$ & $\begin{array}{l}\text { PhD student Cremilda } \\
\text { Barbosa de Souza; PhD } \\
\text { student Teresa Cristina } \\
\text { Monteiro Ferreira }\end{array}$ & $\begin{array}{l}\text { Comparative study of trifluorpromazine } \\
\text { impregnation; Side effects in the use of } \\
\text { derivatives of phenothiazines }\end{array}$ & Jornal do Comércio & $\mathrm{N} / \mathrm{A}$ \\
\hline
\end{tabular}




\begin{tabular}{|c|c|c|c|c|c|}
\hline Date & Event type & Presented by & Theme & Journal/Font & Event place \\
\hline $11 / 05 / 62$ & $\begin{array}{l}\text { Thematic } \\
\text { presentation }\end{array}$ & $\begin{array}{l}\text { Prof. Koelle da Universidade } \\
\text { da Pensivânia }\end{array}$ & Psychopharmacology & Diário de notícias; & IPUB's Amphitheater \\
\hline 20/07/62 & $\begin{array}{l}\text { Thematic } \\
\text { presentation }\end{array}$ & Dr Maxwell Gitelson & The role of anxiety in somatic illnesses & Diário de notícias; & $\mathrm{N} / \mathrm{A}$ \\
\hline 02/08/62 & $\begin{array}{l}\text { Thematic } \\
\text { presentation }\end{array}$ & $\begin{array}{l}\text { PhD student Dirceu de } \\
\text { Santa Rosa; PhD student } \\
\text { José Antônio Couto and } \\
\text { Silva and Icêma de Oliveira; } \\
\text { PhD student Roberto } \\
\text { Bittencourt Martins }\end{array}$ & $\begin{array}{l}\text { Contribution to the study of the } \\
\text { personalities of young drug addicts; } \\
\text { Previous note on urinary excretion } \\
\text { levels of } 17 \text { corticosteroids in the } \\
\text { course of therapeutic administration of } \\
\text { high doses of phenothiazines; The } \\
\text { supernatural in primitive mentality and } \\
\text { schizophrenia }\end{array}$ & Diário de notícias; 0 jornal & IPUB's Amphitheater \\
\hline 24/08/62 & $\begin{array}{l}\text { Thematic } \\
\text { presentation }\end{array}$ & $\begin{array}{l}\text { Dr Valderedo do Ismael } \\
\text { Oliveira; Dr Wilson Lara } \\
\text { Chebadi; DR Oswaldo dos } \\
\text { Santos }\end{array}$ & $\begin{array}{l}\text { Psychoanalysis of Social Institutions; } \\
\text { War in the light of experience with } \\
\text { groups; Indication of occupational } \\
\text { therapeutic activity in Psychiatry }\end{array}$ & Diário de notícias; & IPUB's Amphitheater \\
\hline 04/01/63 & $\begin{array}{l}\text { Bibliography } \\
\text { review }\end{array}$ & $\begin{array}{l}\text { Prof. José Leme Lopes, Dr } \\
\text { Adolpho Hoirisch, Dr Dr } \\
\text { Miriam Escoteguy and } \\
\text { Ulysses Vianna Filho }\end{array}$ & Literature review & & \\
\hline $11 / 01 / 63$ & Clinical case & $\begin{array}{l}\text { Dra Wanda Leme Pereira; } \\
\text { Dr Wilson L Chebabi }\end{array}$ & $\begin{array}{l}\text { Clinical case; Psychotherapy clinical } \\
\text { case }\end{array}$ & & \\
\hline 18/01/63 & $\begin{array}{l}\text { Thematic } \\
\text { presentation }\end{array}$ & $\begin{array}{l}\text { Prof. José Leme Lopes; } \\
\text { Docente Walderedo I. De } \\
\text { Oliveira }\end{array}$ & $\begin{array}{l}\text { About delusional perception; On the } \\
\text { primitive development of the individual }\end{array}$ & & \\
\hline 25/01/63 & Clinical case & $\begin{array}{l}\text { Registrar Paulo Lang; } \\
\text { Registrar Fernando B. } \\
\text { Correa }\end{array}$ & & & \\
\hline 01/03/63 & $\begin{array}{l}\text { Bibliography } \\
\text { review }\end{array}$ & $\begin{array}{l}\text { Prof. José Leme Lopes, Dr } \\
\text { Adolpho Hoirisch }\end{array}$ & & & \\
\hline 08/03/63 & Clinical case & $\begin{array}{l}\text { PhD student Jay Bisker and } \\
\text { Dr Marcello de Paula }\end{array}$ & & & \\
\hline $15 / 03 / 63$ & $\begin{array}{l}\text { Bibliography } \\
\text { review }\end{array}$ & Prof José Leme Lopes & $\begin{array}{l}\text { Synthesis of Binswanger's Mania and } \\
\text { Melancholia book }\end{array}$ & Diário de notícias; & $\mathrm{N} / \mathrm{A}$ \\
\hline 22/03/63 & $\begin{array}{l}\text { Bibliography } \\
\text { review }\end{array}$ & Prof. José Leme Lopes & $\begin{array}{l}\text { Ludwig Binswanger's Mania and } \\
\text { Melancholia book }\end{array}$ & Diário de notícias; & $\mathrm{N} / \mathrm{A}$ \\
\hline 29/03/63 & Clinical case & $\begin{array}{l}\text { Dr Gladstone Parente and } \\
\text { Dra Iza Alpoim da Matta }\end{array}$ & & & \\
\hline 05/04/63 & $\begin{array}{l}\text { Bibliography } \\
\text { review }\end{array}$ & $\begin{array}{l}\text { Prof. José Leme Lopes, Dr } \\
\text { Adolpho Hoirisch, Prof. } \\
\text { Candido Bastos, Dr Portela } \\
\text { Nunes and DR J.C. } \\
\text { Madalena }\end{array}$ & & & \\
\hline $10 / 04 / 63$ & $\begin{array}{l}\text { Thematic } \\
\text { presentation }\end{array}$ & Prof. Eugene B Brody & $\begin{array}{l}\text { Some conceptual and methodological } \\
\text { problems in research on society, culture } \\
\text { and mental illness }\end{array}$ & & \\
\hline $19 / 04 / 63$ & $\begin{array}{l}\text { Thematic } \\
\text { presentation }\end{array}$ & Dr J Jansen & New psychotropic medicines & Correio da Manhã & N/A \\
\hline 26/04/63 & $\begin{array}{l}\text { Thematic } \\
\text { presentation }\end{array}$ & Dr M. Cabalero Goas & $\begin{array}{l}\text { Humor and delusional perception; } \\
\text { Concept of schizophrenic psychosis }\end{array}$ & Correio da Manhã & $\mathrm{N} / \mathrm{A}$ \\
\hline 03/05/63 & Clinical case & $\begin{array}{l}\text { Dra Ana Elisa Mercadante, } \\
\text { Dra Iza Alpoim Matta and } \\
\text { Dr Gladstone Parente }\end{array}$ & & & \\
\hline $10 / 05 / 63$ & $\begin{array}{l}\text { Bibliography } \\
\text { review }\end{array}$ & $\begin{array}{l}\text { Dra Innocência Kranner } \\
\text { Frank, Dr Caruso Madalena, } \\
\text { Dr Adolpho Hoirisch, Prof } \\
\text { Leme Lopes and Dra Ana } \\
\text { Elisa Mercadante }\end{array}$ & & & \\
\hline
\end{tabular}




\begin{tabular}{|c|c|c|c|c|c|}
\hline Date & Event type & Presented by & Theme & Journal/Font & Event place \\
\hline $17 / 05 / 63$ & Clinical case & $\begin{array}{l}\text { Dr Jacob Azulay, Dra } \\
\text { Miriam Andraus, Acad Lúcia } \\
\text { Costa }\end{array}$ & & & \\
\hline $18 / 05 / 63$ & $\begin{array}{l}\text { Thematic } \\
\text { presentation }\end{array}$ & Prof José Leme Lopes & About delirant perception & Correio da Manhã & $\mathrm{N} / \mathrm{A}$ \\
\hline 24/05/63 & $\begin{array}{l}\text { Thematic } \\
\text { presentation }\end{array}$ & $\begin{array}{l}\text { Dr. José Caruso Madalena; } \\
\text { Dr Wilsom de Lyra Chebabi }\end{array}$ & $\begin{array}{l}\text { Benzodiazepines - new class of } \\
\text { anticonvulsant substances; Outline of } \\
\text { the group therapeutic situation }\end{array}$ & Diário de notícias; Correio da Manhã & $\mathrm{N} / \mathrm{A}$ \\
\hline $31 / 05 / 63$ & Clinical case & $\begin{array}{l}\text { Dra Miriam Andraus and } \\
\text { PhD student Lucia Costa }\end{array}$ & & & \\
\hline 07/06/63 & $\begin{array}{l}\text { Bibliography } \\
\text { review }\end{array}$ & $\begin{array}{l}\text { Prof Jose Leme Lopes, Dr } \\
\text { Rawlinson Prestes Lemos } \\
\text { and Dra Miriam Escoteguy, } \\
\text { PhD student Lucia Costa, } \\
\text { Dr Wilson Chebabi, Dr } \\
\text { Henrique de Novaes Filho } \\
\text { and Dr J.C. Madalena }\end{array}$ & & Homage ao papa João XXIII & \\
\hline $14 / 06 / 63$ & $\begin{array}{l}\text { Thematic } \\
\text { presentation }\end{array}$ & $\begin{array}{l}\text { Prof. Jurandyr Manfredini; } \\
\text { Docente Walderedo Ismael } \\
\text { Oliveira; Doutor E. Portella } \\
\text { Nunes Filho; Prof Henrique } \\
\text { Roxo }\end{array}$ & $\begin{array}{l}\text { Rorschac and Perception; Racism and } \\
\text { Therapeutic Groups; Introjection, } \\
\text { projection and reality }\end{array}$ & Correio da Manhã; Diario de Notícias & $\mathrm{N} / \mathrm{A}$ \\
\hline 21/06/63 & Clinical case & $\begin{array}{l}\text { PhD student Lucia Costa } \\
\text { and PhD student Franisco } \\
\text { Soerdenberg }\end{array}$ & & & \\
\hline 28/06/63 & $\begin{array}{l}\text { Bibliography } \\
\text { review }\end{array}$ & $\begin{array}{l}\text { Dr José Candido Bastos, Dr } \\
\text { Adolpho Hoirisch, Dr Wilson } \\
\text { Chebabi, Dr Henrique } \\
\text { Novaes Filho, Dr Mauricio } \\
\text { Schiller }\end{array}$ & & & \\
\hline 05/07/63 & Clinical case & $\begin{array}{l}\text { Dr Vidal Dutra Filho and Dra } \\
\text { Miriam Escosteguy }\end{array}$ & & & \\
\hline $12 / 07 / 63$ & $\begin{array}{l}\text { Thematic } \\
\text { presentation }\end{array}$ & Dr Luiz Cerqueira & $\begin{array}{l}\text { The fate of painting in occupational } \\
\text { therapy }\end{array}$ & & \\
\hline $19 / 07 / 63$ & Clinical case & $\begin{array}{l}\text { Dr Vidal Dutra Filho and Dra } \\
\text { Ana Elisa Mercadante }\end{array}$ & & & \\
\hline 26/07/63 & $\begin{array}{l}\text { Bibliography } \\
\text { review }\end{array}$ & $\begin{array}{l}\text { Dra Ana Elisa Mercadante, } \\
\text { Dr Mauricio Schiller, Dr } \\
\text { Ulysses Vianna Filho and } \\
\text { Dra Innocencia Kranner } \\
\text { Frank }\end{array}$ & & & \\
\hline 02/08/63 & Clinical case & $\begin{array}{l}\text { Dr Miriam Andraus, Acad } \\
\text { Dias Campos, PhD student } \\
\text { Joelza Figueiredo }\end{array}$ & & & \\
\hline 09/08/63 & $\begin{array}{l}\text { Thematic } \\
\text { presentation }\end{array}$ & $\begin{array}{l}\text { Prof Jose Leme Lopes; Dr } \\
\text { Wilson Chebabi; Dr } \\
\text { Henrique de Novaes Filho }\end{array}$ & $\begin{array}{l}\text { Group psychotherapy; Capitalism and } \\
\text { dependence in the therapeutic group }\end{array}$ & & \\
\hline $16 / 08 / 63$ & Clinical case & $\begin{array}{l}\text { Dr Gladstone Parente and } \\
\text { Acad Moisés Groismann }\end{array}$ & & & \\
\hline 23/08/63 & $\begin{array}{l}\text { Bibliography } \\
\text { review }\end{array}$ & $\begin{array}{l}\text { Dr Adolpho Hoirisch, Dr } \\
\text { Caruso Madalena, Dr } \\
\text { Rawlinson P Lemos, Dra } \\
\text { Wanda L. Pereira, Dr } \\
\text { Mauricio Schiller and Dr } \\
\text { Ulysses Viana Filho }\end{array}$ & & & \\
\hline $30 / 08 / 63$ & Clinical case & $\begin{array}{l}\text { Dra Iza Alphim da Matta } \\
\text { and PhD student Roberto } \\
\text { Quilelli }\end{array}$ & & Correio da Manhã & $\mathrm{N} / \mathrm{A}$ \\
\hline
\end{tabular}




\begin{tabular}{|c|c|c|c|c|c|}
\hline Date & Event type & Presented by & Theme & Journal/Font & Event place \\
\hline 06/09/63 & $\begin{array}{l}\text { Thematic } \\
\text { presentation }\end{array}$ & $\begin{array}{l}\text { PhD student Roberto Quilelli } \\
\text { Correa; Dr Ulysses Vianna } \\
\text { Filho }\end{array}$ & $\begin{array}{l}\text { Atividade musical em terapêutica } \\
\text { ocupacional; Pesquisa } \\
\text { eletroencefalográfica em } \\
\text { psicosesMusical activity in occupational } \\
\text { therapy; Electroencephalographic } \\
\text { research in psychoses }\end{array}$ & & \\
\hline $13 / 09 / 63$ & Clinical case & $\begin{array}{l}\text { Dr Raulito Gomes and Dr } \\
\text { Henrique de Novaes Filho }\end{array}$ & & & \\
\hline 20/09/63 & $\begin{array}{l}\text { Bibliography } \\
\text { review }\end{array}$ & $\begin{array}{l}\text { Dra Icema Oliveira, Dr } \\
\text { Dirceu Santa Rosa, Dr } \\
\text { Jayme Bisker, Dr jacob } \\
\text { Azulay }\end{array}$ & & & \\
\hline 25/09/63 & Homage & $\begin{array}{l}\text { Prof. Decio Soares de } \\
\text { Souza, Dra Marialzira } \\
\text { Perestrello and Dra Mara } \\
\text { Salvini de Souza }\end{array}$ & $\begin{array}{l}\text { Symposium of the 10th Anniversary of } \\
\text { the Child Guidance Clinic }\end{array}$ & & \\
\hline 27/09/63 & Homage & $\begin{array}{l}\text { Dra Ana Elisa Mercadante, } \\
\text { Dr Vidal Dutra Filho, Da } \\
\text { Jovita Madeira Martins, Da } \\
\text { Julia Maria de Almeida } \\
\text { Chermont and Dr Renato } \\
\text { Nunes Esteves }\end{array}$ & $\begin{array}{l}\text { Symposium of the 10th Anniversary of } \\
\text { the Child Guidance Clinic }\end{array}$ & & \\
\hline 04/10/63 & $\begin{array}{l}\text { Thematic } \\
\text { presentation }\end{array}$ & Dr Luis Ribeiro & Psychiatrist as a hospital administrator. & & \\
\hline $11 / 10 / 63$ & Clinical case & $\begin{array}{l}\text { PhD student Roberto Quilelli } \\
\text { Correa; Dra Miriam Andraus }\end{array}$ & & & \\
\hline 18/10/63 & $\begin{array}{l}\text { Bibliography } \\
\text { review }\end{array}$ & $\begin{array}{l}\text { Dr Adolpho Hoirisch, Dr } \\
\text { Caruso Madalena, Dr Jacob } \\
\text { Azulay and Dr Wilson } \\
\text { Chebabi }\end{array}$ & & & \\
\hline 25/10/63 & Clinical case & $\begin{array}{l}\text { PhD student Lucia Costa } \\
\text { and Dra Jucedy N. Ribeiro }\end{array}$ & & & \\
\hline 08/11/63 & $\begin{array}{l}\text { Thematic } \\
\text { presentation }\end{array}$ & Dr Alvaro Lima Costa & Dysmetabolic oligophrenias & & \\
\hline 08/11/63 & Clinical case & $\begin{array}{l}\text { Dr Santana and Dr Luiz } \\
\text { Cerqueira }\end{array}$ & & & \\
\hline 29/11/63 & Homage & $\begin{array}{l}\text { Dr Walderedo I de Oliveira; } \\
\text { Dr Alvaro Lima Costa }\end{array}$ & $\begin{array}{l}\text { Homage to Dr Portella Nunes Filho for } \\
\text { the title of Full Professor; Dysmetabolic } \\
\text { oligophrenias }\end{array}$ & Jornal do Brasil & $\mathrm{N} / \mathrm{A}$ \\
\hline 06/12/63 & Clinical case & $\begin{array}{l}\text { Dra Ana Elisa Mercadante, } \\
\text { Dra Mara Salvini de Souza }\end{array}$ & & & \\
\hline $13 / 12 / 63$ & $\begin{array}{l}\text { Bibliography } \\
\text { review }\end{array}$ & $\begin{array}{l}\text { Dr Rawlinson Prestes } \\
\text { Lemos, Dr Mauricio Schiller, } \\
\text { Dr Jacob Azulay, Dr Wilson } \\
\text { Lyra Chebabi, Dr José } \\
\text { Candido Bastos }\end{array}$ & & & \\
\hline 20/12/63 & Clinical case & $\begin{array}{l}\text { Dra Joelza Figueiredo da } \\
\text { Silvam Dr Dirceu Santa } \\
\text { Rosa }\end{array}$ & & & \\
\hline 27/12/63 & $\begin{array}{l}\text { Thematic } \\
\text { presentation }\end{array}$ & $\begin{array}{l}\text { Prof Jose Leme Lopes; Dr } \\
\text { Walderedo I de Oliveira }\end{array}$ & $\begin{array}{l}\text { Farewell to Interns and Registrars; } \\
\text { Study of the evolution of the analysis of } \\
\text { a case of schizophrenia }\end{array}$ & & \\
\hline 08/05/64 & Clinical case & & Psychoneurosis cases & Correio da Manhã & $\mathrm{N} / \mathrm{A}$ \\
\hline $15 / 05 / 64$ & $\begin{array}{l}\text { Bibliography } \\
\text { review }\end{array}$ & $\begin{array}{l}\text { Dr Vidal Dutra Filho, Marcio } \\
\text { Pena Ferreira, Ulisses Viana } \\
\text { Filho, Mara Salvini de Souza }\end{array}$ & & Jornal do Brasil & $\mathrm{N} / \mathrm{A}$ \\
\hline
\end{tabular}




\begin{tabular}{|c|c|c|c|c|c|}
\hline Date & Event type & Presented by & Theme & Journal/Font & Event place \\
\hline 29/05/64 & $\begin{array}{l}\text { Thematic } \\
\text { presentation }\end{array}$ & $\begin{array}{l}\text { Prof José Leme Lopes and } \\
\text { Dr Ulisses Viana Filho; } \\
\text { Dr Luis Cerqueira and } \\
\text { Dr Oswaldo dos Santos }\end{array}$ & $\begin{array}{l}\text { Carbon monoxide poisoning - } \\
\text { schizomorphic syndrome - } \\
\text { electroencephalographic evolution; The } \\
\text { influence of neuroleptics on artistic } \\
\text { production }\end{array}$ & Diário de notícias; & $\mathrm{N} / \mathrm{A}$ \\
\hline $16 / 10 / 64$ & $\begin{array}{l}\text { Bibliography } \\
\text { review }\end{array}$ & $\begin{array}{l}\text { Dr Dirceu Santa Rosa; } \\
\text { Dr Roberto Correa; Dr José } \\
\text { Caruso Madalena; Dra } \\
\text { Wania Lopes Cançado }\end{array}$ & & Correio da Manhã & $\mathrm{N} / \mathrm{A}$ \\
\hline 06/11/64 & Clinical case & $\begin{array}{l}\text { Dr Alberto Tavares and Dr } \\
\text { Nei de Burlamarque de } \\
\text { Alvarenga }\end{array}$ & & Diário de notícias; & $\mathrm{N} / \mathrm{A}$ \\
\hline 19/01/65 & $\begin{array}{l}\text { Thematic } \\
\text { presentation }\end{array}$ & $\begin{array}{l}\text { Dr Romildo Bueno; Ulisses } \\
\text { Viana Filho; Osvaldo } \\
\text { Barbosa }\end{array}$ & $\begin{array}{l}\text { Observation about triperidol and } \\
\text { tegretol }\end{array}$ & Diário de notícias; & $\mathrm{N} / \mathrm{A}$ \\
\hline 19/03/65 & $\begin{array}{l}\text { Bibliography } \\
\text { review }\end{array}$ & $\begin{array}{l}\text { Dr Jorge de Medeiros and } \\
\text { Albuquerque; Raulito } \\
\text { Gomes; Mauricio B Schiller; } \\
\text { William Asmar; Wilson L. } \\
\text { Chebabi }\end{array}$ & & Diário de notícias; & $\mathrm{N} / \mathrm{A}$ \\
\hline $30 / 04 / 65$ & $\begin{array}{l}\text { Bibliography } \\
\text { review }\end{array}$ & $\begin{array}{l}\text { Dr Isa Alpoim da Mata, } \\
\text { Esutachio Portella Nunes } \\
\text { Filho, José Candido Bastos } \\
\text { and Luis Cerqueira }\end{array}$ & & Diário de notícias; & $\mathrm{N} / \mathrm{A}$ \\
\hline $14 / 05 / 65$ & $\begin{array}{l}\text { Thematic } \\
\text { presentation }\end{array}$ & Dr Paulo Dias Correia & $\begin{array}{l}\text { Group dynamics and the petty } \\
\text { bourgeois }\end{array}$ & Jornal do Brasil; D0 & $\mathrm{N} / \mathrm{A}$ \\
\hline 04/06/65 & $\begin{array}{l}\text { Bibliography } \\
\text { review }\end{array}$ & $\begin{array}{l}\text { Dr Walfredo Ismael de } \\
\text { Oliveira; Vanda Leme } \\
\text { Pereira; Inocência K. Frank; } \\
\text { Adolfo Hoirisch }\end{array}$ & & Jornal do Brasil & $\mathrm{N} / \mathrm{A}$ \\
\hline 09/07/65 & $\begin{array}{l}\text { Thematic } \\
\text { presentation }\end{array}$ & Prof Eugene B. Brody & $\begin{array}{l}\text { Cultural Exclusion: An Encouraging } \\
\text { Societal Defense of Individual Illness }\end{array}$ & Diário de notícias; & IPUB's Amphitheater \\
\hline 02/09/65 & $\begin{array}{l}\text { Bibliography } \\
\text { review }\end{array}$ & $\begin{array}{l}\text { Dr Miriam Caminha } \\
\text { Escostegui; Dra Ana Elisa } \\
\text { Viana Mercadante; Marcio } \\
\text { P. Pereira; Rewlinson P. } \\
\text { Lemos; Henrique de Novais } \\
\text { Filho; J.C. Madalena and } \\
\text { Edmundo Haes }\end{array}$ & & Diário de notícias; & $\mathrm{N} / \mathrm{A}$ \\
\hline $17 / 09 / 65$ & $\begin{array}{l}\text { Thematic } \\
\text { presentation }\end{array}$ & Dr Stanislau Krynski & Dysmetabolic oligophrenias & Diário de notícias; & IPUB's Amphitheater \\
\hline 24/09/65 & $\begin{array}{l}\text { Thematic } \\
\text { presentation }\end{array}$ & $\begin{array}{l}\text { Dr Valderedo do Ismael } \\
\text { Oliveira; Maria Luisa Pinto } \\
\text { and Davi Jacó Azulay }\end{array}$ & $\begin{array}{l}\text { Basic anxieties and fantasies in the } \\
\text { therapeutic group }\end{array}$ & Diário de notícias; & $\mathrm{N} / \mathrm{A}$ \\
\hline 08/10/65 & $\begin{array}{l}\text { Thematic } \\
\text { presentation }\end{array}$ & $\begin{array}{l}\text { Dr Isa Alpoim da Mata and } \\
\text { Wilson Lira Chebabi }\end{array}$ & The melancholic syndrome & Diário de notícias; & $\mathrm{N} / \mathrm{A}$ \\
\hline 22/10/65 & $\begin{array}{l}\text { Thematic } \\
\text { presentation }\end{array}$ & Dr Jacob David Azulay & Psychopathology of conversion & Diário de notícias; & $\mathrm{N} / \mathrm{A}$ \\
\hline $12 / 11 / 65$ & $\begin{array}{l}\text { Thematic } \\
\text { presentation }\end{array}$ & $\begin{array}{l}\text { Dr Galina Schneider and } \\
\text { Washington Loyello }\end{array}$ & Despersonalization syndrome & Diário de notícias; & $\mathrm{N} / \mathrm{A}$ \\
\hline $12 / 01 / 66$ & $\begin{array}{l}\text { Thematic } \\
\text { presentation }\end{array}$ & Prof Fernandes da Fonseca & Social Psychiatry & Diário de notícias; & $\mathrm{N} / \mathrm{A}$ \\
\hline 18/03/66 & $\begin{array}{l}\text { Thematic } \\
\text { presentation }\end{array}$ & $\begin{array}{l}\text { Prof Leme Lopes and } \\
\text { William Asmar }\end{array}$ & $\begin{array}{l}\text { Introduction to psychiatry by Machado } \\
\text { de Assis in a commentary on the short } \\
\text { story "the alienist"; Contributions to the } \\
\text { study of object relation }\end{array}$ & O Jornal; Diário de notícias & $\mathrm{N} / \mathrm{A}$ \\
\hline 01/04/66 & $\begin{array}{l}\text { Bibliography } \\
\text { review }\end{array}$ & $\begin{array}{l}\text { Dr Vidal Dutra Filho, Ulisses } \\
\text { Viana Filho, Mara Salvini de } \\
\text { Souza }\end{array}$ & & Jornal do Comércio & IPUB's head office \\
\hline
\end{tabular}




\begin{tabular}{|c|c|c|c|c|c|}
\hline Date & Event type & Presented by & Theme & Journal/Font & Event place \\
\hline 29/04/66 & $\begin{array}{l}\text { Thematic } \\
\text { presentation }\end{array}$ & Prof Emanuel Carneiro Leão & The method of existential analysis & Correio da Manhã & $\mathrm{N} / \mathrm{A}$ \\
\hline 28/05/66 & Clinical case & $\begin{array}{l}\text { Dr Ana Elisa Viana } \\
\text { Mercadante and Vidal Dutra } \\
\text { Filho }\end{array}$ & & & $\mathrm{N} / \mathrm{A}$ \\
\hline 19/06/66 & $\begin{array}{l}\text { Thematic } \\
\text { presentation }\end{array}$ & $\begin{array}{l}\text { Dr Wilson Lira Chebabi; Dr } \\
\text { Cerqueira, Fabio Lacombe } \\
\text { and Nei Martins }\end{array}$ & $\begin{array}{l}\text { Study of the hallucinatory world; Report } \\
\text { on the IV journey of Psychiatry in Rio } \\
\text { Grande do Sul }\end{array}$ & & $\mathrm{N} / \mathrm{A}$ \\
\hline 18/07/66 & Clinical case & $\begin{array}{l}\text { Acad Joaqueim Amélio } \\
\text { Couto and Fabio Penna } \\
\text { Lacombe }\end{array}$ & & & $\mathrm{N} / \mathrm{A}$ \\
\hline 27/08/66 & $\begin{array}{l}\text { Thematic } \\
\text { presentation }\end{array}$ & $\begin{array}{l}\text { Dr Wilson Lira Chebabi; } \\
\text { Ulisses Viana Filho }\end{array}$ & $\begin{array}{l}\text { Sleep and dream from a } \\
\text { neurophysiological point of view; Sleep } \\
\text { and dream from a psychological point } \\
\text { of view; Repercussions of the } \\
\text { discussions of the international } \\
\text { congress of psychiatry }\end{array}$ & & $\mathrm{N} / \mathrm{A}$ \\
\hline $11 / 11 / 66$ & $\begin{array}{l}\text { Bibliography } \\
\text { review }\end{array}$ & $\begin{array}{l}\text { Dr Vidal Dutra Filho, Marcio } \\
\text { Pena Ferreira, Mara Salvini } \\
\text { de Souza, Iza Alpeim da } \\
\text { Matta }\end{array}$ & & Correio da Manhã & $\mathrm{N} / \mathrm{A}$ \\
\hline $10 / 12 / 66$ & $\begin{array}{l}\text { Thematic } \\
\text { presentation }\end{array}$ & Prof Paulo da Silca Lacaz & $\begin{array}{l}\text { Biochemical conceptualization of } \\
\text { schizophrenia }\end{array}$ & Diário de notícias; & N/A \\
\hline $19 / 05 / 67$ & $\begin{array}{l}\text { Thematic } \\
\text { presentation }\end{array}$ & Dr José Luis Gonzalez & Group Psychoanalysis in Mexico & Diário de notícias; & $\mathrm{N} / \mathrm{A}$ \\
\hline 28/07/67 & $\begin{array}{l}\text { Bibliography } \\
\text { review }\end{array}$ & $\begin{array}{l}\text { Regina Helena Taeola Terra, } \\
\text { Roberto Kedhi, Adalberto } \\
\text { Rodrigues da Silva, Antonio } \\
\text { Osvaldo de Aquino, Jaques } \\
\text { Vieira Engel, Fabio Pena } \\
\text { Lacombe, Leon Capeler, } \\
\text { Roberto Amorim Robalinho } \\
\text { de Oliveira Cavalcanti, } \\
\text { Andre Faria de Azevedo } \\
\text { Carneiro, Antonio lounis, } \\
\text { José Carlos Zanin, Luis } \\
\text { Sergio Lobianco, Giorgio } \\
\text { Troto }\end{array}$ & & & N/A \\
\hline 04/08/67 & Clinical case & & & Diário de notícias; & N/A \\
\hline $11 / 08 / 67$ & & $\begin{array}{l}\text { Dr Cerqueira and Dr } \\
\text { Hamilton Siqueira }\end{array}$ & & Diário de notícias; & $\mathrm{N} / \mathrm{A}$ \\
\hline 15/09/67 & $\begin{array}{l}\text { Thematic } \\
\text { presentation }\end{array}$ & $\begin{array}{l}\text { Prof. Decio Soares de } \\
\text { Souza; }\end{array}$ & Doctor patient relationship & Jornal do Brasil; diario de noticias & $\mathrm{N} / \mathrm{A}$ \\
\hline $18 / 10 / 67$ & $\begin{array}{l}\text { Thematic } \\
\text { presentation }\end{array}$ & Prof José Leme Lopes & $\begin{array}{l}\text { General review of the Porto Alegre } \\
\text { Psychiatry Congress; Community } \\
\text { Mental Health and Psychiatry }\end{array}$ & Jornal do Brasil & $\mathrm{N} / \mathrm{A}$ \\
\hline 27/10/67 & $\begin{array}{l}\text { Thematic } \\
\text { presentation }\end{array}$ & Prof. Waldredo I. De Oliveira & $\begin{array}{l}\text { Transference, interpretation and } \\
\text { vicissitudes of fantasy; Report on the } \\
\text { Brazilian Congress of Neurology, } \\
\text { Psychiatry and Mental Hygiene of Porto } \\
\text { Alegre }\end{array}$ & Jornal do Brasil & $\mathrm{N} / \mathrm{A}$ \\
\hline 28/11/67 & $\begin{array}{l}\text { Thematic } \\
\text { presentation }\end{array}$ & Dr Wilson Chebabi; & $\begin{array}{l}\text { Introduction to the psychodynamics of } \\
\text { crime }\end{array}$ & Jornal do Brasil & $\mathrm{N} / \mathrm{A}$ \\
\hline $12 / 01 / 68$ & $\begin{array}{l}\text { Thematic } \\
\text { presentation }\end{array}$ & Dr Fabio Leite Lobo & $\begin{array}{l}\text { Contributions of Fairbairn to } \\
\text { psychoanalysis }\end{array}$ & Jornal do Brasil; correio da manhã & $\mathrm{N} / \mathrm{A}$ \\
\hline 13/04/68 & $\begin{array}{l}\text { Thematic } \\
\text { presentation }\end{array}$ & $\begin{array}{l}\text { Dr Ney Couto Marinho and } \\
\text { Roberto Quileli Correia }\end{array}$ & $\begin{array}{l}\text { Successes and limitations of } \\
\text { occupational therapy in chronic patients }\end{array}$ & Correio da Manhã & $\mathrm{N} / \mathrm{A}$ \\
\hline
\end{tabular}




\begin{tabular}{|c|c|c|c|c|c|}
\hline Date & Event type & Presented by & Theme & Journal/Font & Event place \\
\hline $31 / 05 / 68$ & $\begin{array}{l}\text { Thematic } \\
\text { presentation }\end{array}$ & $\begin{array}{l}\text { Walderedo I de Oliveira; J. } \\
\text { Caruso Madalena }\end{array}$ & $\begin{array}{l}\text { Transference, Interpretation and } \\
\text { Vicissitudes of Fantasy; Drug Update in } \\
\text { Child Psychiatry }\end{array}$ & $\begin{array}{l}\text { Sessão conjunta com a Associação } \\
\text { Psiquiatrica do Rio de Janeiro }\end{array}$ & \\
\hline 01/06/68 & $\begin{array}{l}\text { Thematic } \\
\text { presentation }\end{array}$ & $\begin{array}{l}\text { José Leme Lopes; J. Garcia } \\
\text { Alves; Sérgio Lobianco }\end{array}$ & $\begin{array}{l}\text { Concept of mental illness according to } \\
\text { Kurt Schneider; Psychopathic } \\
\text { personalities according to Kurt } \\
\text { Schneider; Primary and secondary } \\
\text { symptoms in schizophrenia; Homage to } \\
\text { Kurt Schneider }\end{array}$ & & \\
\hline 12/07/68 & $\begin{array}{l}\text { Thematic } \\
\text { presentation }\end{array}$ & John S Peck & $\begin{array}{l}\text { Aspects of the formation of group } \\
\text { therapists in the USA }\end{array}$ & & \\
\hline 27/12/68 & Homage & & $\begin{array}{l}\text { End of the Interns and Registrars School } \\
\text { Year }\end{array}$ & & \\
\hline 22/07/69 & $\begin{array}{l}\text { Thematic } \\
\text { presentation }\end{array}$ & Prof Leme Lopes & $\begin{array}{l}\text { Impressions of a visit to a European } \\
\text { psychiatric clinic }\end{array}$ & Jornal do Comércio & $\mathrm{N} / \mathrm{A}$ \\
\hline $25 / 05 / 73$ & $\begin{array}{l}\text { Thematic } \\
\text { presentation }\end{array}$ & Prof Caballero Goas & $\begin{array}{l}\text { State of the art about psychiatric } \\
\text { investigation }\end{array}$ & Correio da Manhã & $N / A$ \\
\hline $31 / 08 / 73$ & Homage & $\begin{array}{l}\text { Prof Portella Nunes, Prof } \\
\text { Neves Manta, Pres. Leme } \\
\text { Lopes }\end{array}$ & $\begin{array}{l}\text { Teaching of psychiatry and medical } \\
\text { psychology; Homage to Pro Neves } \\
\text { Manta }\end{array}$ & Correio da Manhã & $\mathrm{N} / \mathrm{A}$ \\
\hline 18/04/75 & $\begin{array}{l}\text { Thematic } \\
\text { presentation }\end{array}$ & & $\begin{array}{l}\text { A critical evaluation of the } 1974 \\
\text { specialization course in psychiatry }\end{array}$ & & \\
\hline 02/05/75 & $\begin{array}{l}\text { Thematic } \\
\text { presentation }\end{array}$ & Dr Theodor Lovenkron & Psychiatric Epidemiology & & \\
\hline 09/05/75 & $\begin{array}{l}\text { Bibliography } \\
\text { review }\end{array}$ & $\begin{array}{l}\text { Edson Duarte; Laurentino } \\
\text { Barbosa Lopes }\end{array}$ & & & \\
\hline $30 / 05 / 75$ & $\begin{array}{l}\text { Thematic } \\
\text { presentation }\end{array}$ & Profa Neuma Aguiar & $\begin{array}{l}\text { Psychiatric Institution, community and } \\
\text { family }\end{array}$ & & \\
\hline 06/06/75 & $\begin{array}{l}\text { Thematic } \\
\text { presentation }\end{array}$ & Profa Ruth Joffily Dias & $\begin{array}{l}\text { Therapeutics, experimentation and } \\
\text { responsibility; George Canguilhem text } \\
\text { reading }\end{array}$ & & \\
\hline $27 / 06 / 75$ & $\begin{array}{l}\text { Thematic } \\
\text { presentation }\end{array}$ & $\begin{array}{l}\text { Profa Rosiane Joseph } \\
\text { Perellberg }\end{array}$ & The psychiatric opinion "deviation case" & & \\
\hline $30 / 08 / 75$ & $\begin{array}{l}\text { Bibliography } \\
\text { review }\end{array}$ & Luis Fernando Mello & & & \\
\hline 26/09/75 & $\begin{array}{l}\text { Thematic } \\
\text { presentation }\end{array}$ & Prof Alonso Fernandes & Depression clinics & & \\
\hline $10 / 11 / 75$ & $\begin{array}{l}\text { Thematic } \\
\text { presentation }\end{array}$ & Prof dr J Romildo Bueno & Daily dose of neuroleptics & & \\
\hline 20/11/75 & Homage & & $\begin{array}{l}\text { Homage to Prof Leme Lopes; } 50 \text { years } \\
\text { symposium of psychiatry }\end{array}$ & jornal dos sports & IPUB's auditorium \\
\hline 09/01/76 & $\begin{array}{l}\text { Thematic } \\
\text { presentation }\end{array}$ & Eustachio Portella Nunes & $\begin{array}{l}\text { The sense of technique in } \\
\text { psychoanalysis }\end{array}$ & & \\
\hline 23/01/76 & Clinical case & $\begin{array}{l}\text { Jorge Adelino, Nilza } \\
\text { Fernandes, Julia Chermont, } \\
\text { José Onildo }\end{array}$ & & & \\
\hline 19/03/76 & $\begin{array}{l}\text { Thematic } \\
\text { presentation }\end{array}$ & Wilson de Lyra Chebabi & $\begin{array}{l}\text { The importance of some middle age } \\
\text { themes for clinical practice }\end{array}$ & & \\
\hline 16/07/76 & Clinical case & $\begin{array}{l}\text { Angela Ribeiro G da Silva, } \\
\text { Carlos Edson Duarte, Sara } \\
\text { Botner }\end{array}$ & & & \\
\hline $30 / 07 / 76$ & Clinical case & $\begin{array}{l}\text { Jorge Adelino, Sonia Alves, } \\
\text { Ana Carolina Clementino, } \\
\text { Margarida Assad, Silvia } \\
\text { Regina Parça }\end{array}$ & & & \\
\hline $13 / 08 / 76$ & $\begin{array}{l}\text { Thematic } \\
\text { presentation }\end{array}$ & Carlos Henrique Escobar & Psychoanalysis and Ideology & & \\
\hline
\end{tabular}




\begin{tabular}{|c|c|c|c|c|c|}
\hline Date & Event type & Presented by & Theme & Journal/Font & Event place \\
\hline 20/08/76 & $\begin{array}{l}\text { Thematic } \\
\text { presentation }\end{array}$ & Sidonie Melher & $\begin{array}{l}\text { Models of La Teoria de Los Instintos in } \\
\text { Freud }\end{array}$ & Probably held in Spanish & \\
\hline 27/08/76 & $\begin{array}{l}\text { Masters } \\
\text { dissertation }\end{array}$ & Carlos Edson Duarte & Community and prevention & & \\
\hline $17 / 09 / 76$ & $\begin{array}{l}\text { Thematic } \\
\text { presentation }\end{array}$ & $\begin{array}{l}\text { Francisco Araujo, Munira } \\
\text { Alex, Sonia Teresa Santos }\end{array}$ & Psychotherapeutic care in an institution & & \\
\hline 09/07/87 & Homage & & $\begin{array}{l}\text { Homage to Walderedo Ismael de } \\
\text { Oliveira }\end{array}$ & Jornal do Brasil & $\mathrm{N} / \mathrm{A}$ \\
\hline $11 / 03 / 94$ & $\begin{array}{l}\text { PhD thesis } \\
\text { defense }\end{array}$ & Dra Silvia Rodrigues Jardim & $\begin{array}{l}\text { Work process and psychic suffering: the } \\
\text { case of Rio metro pilots }\end{array}$ & & \\
\hline $18 / 03 / 94$ & $\begin{array}{l}\text { Thematic } \\
\text { presentation }\end{array}$ & $\begin{array}{l}\text { Prof João Ferreira da Silva } \\
\text { Filho and diretores }\end{array}$ & $\begin{array}{l}\text { Assistance, Teaching and Research at } \\
\text { IPUB }\end{array}$ & & \\
\hline 25/03/94 & $\begin{array}{l}\text { Thematic } \\
\text { presentation }\end{array}$ & $\begin{array}{l}\text { Prof Eustachio Portella } \\
\text { Nunes Filho, Prof Adolpho } \\
\text { Hoirisch, Prof Jorge Alberto } \\
\text { Costa and Silva }\end{array}$ & $\begin{array}{l}\text { Psychiatry in the XXlth century; ABP/ } \\
\text { APERJ meeting }\end{array}$ & & \\
\hline 08/04/94 & $\begin{array}{l}\text { Thematic } \\
\text { presentation }\end{array}$ & $\begin{array}{l}\text { Dr Manoel Olavo Teixeira, } \\
\text { Psicóloga Maria Paula } \\
\text { Gomes }\end{array}$ & $\begin{array}{l}\text { Mental health care in the city of Angra } \\
\text { dos Reis }\end{array}$ & & \\
\hline $15 / 04 / 94$ & $\begin{array}{l}\text { Thematic } \\
\text { presentation }\end{array}$ & $\begin{array}{l}\text { Dra Clara Helena Portella, } \\
\text { Dr Marcos Gebara, Psic } \\
\text { Bernard Rangé }\end{array}$ & Psychotherapies & ABP/APERJ & \\
\hline 29/04/94 & $\begin{array}{l}\text { Thematic } \\
\text { presentation }\end{array}$ & Dr José Candido Bastos & Historical basis of psychoanalysis & & \\
\hline 06/05/94 & $\begin{array}{l}\text { Institutional } \\
\text { Congress }\end{array}$ & & & & \\
\hline $13 / 05 / 94$ & $\begin{array}{l}\text { Thematic } \\
\text { presentation }\end{array}$ & $\begin{array}{l}\text { Dr Julio Verztman and } \\
\text { equipe }\end{array}$ & $\begin{array}{l}\text { New mental health paradigms in the } \\
\text { Carlos Antônio da Silva health center } \\
\text { - Niterói }\end{array}$ & & \\
\hline 20/05/94 & Homage & $\begin{array}{l}\text { Prof Eustachio Portella } \\
\text { Nunes Filho, Prof Adolpho } \\
\text { Hoirisch }\end{array}$ & & & \\
\hline 27/05/94 & $\begin{array}{l}\text { Thematic } \\
\text { presentation }\end{array}$ & $\begin{array}{l}\text { Dra Maria Tavares } \\
\text { Cavalcanti, Dra Tania } \\
\text { Marins and equipe com } \\
\text { pacientes do projeto } \\
\text { portaria }\end{array}$ & $\begin{array}{l}\text { Psychosis and work: project ordinance } \\
\text { at the Jurujuba State Psychiatric } \\
\text { Hospital }\end{array}$ & & \\
\hline $10 / 06 / 94$ & $\begin{array}{l}\text { Thematic } \\
\text { presentation }\end{array}$ & $\begin{array}{l}\text { Dr José Alberto Zusman } \\
\text { and equipe }\end{array}$ & $\begin{array}{l}\text { Partial Hospitalization at IPUB: a work } \\
\text { philosophy }\end{array}$ & & \\
\hline $17 / 06 / 94$ & $\begin{array}{l}\text { Thematic } \\
\text { presentation }\end{array}$ & $\begin{array}{l}\text { Dr José Alberto Zusman, Dr } \\
\text { Marco Aurelio Jorge, Dra } \\
\text { Guaraciny Vieira, Psic Elaine } \\
\text { Sari }\end{array}$ & $\begin{array}{l}\text { Permanent mental health day care } \\
\text { forum }\end{array}$ & ABP/APERJ & \\
\hline 24/06/94 & $\begin{array}{l}\text { Thematic } \\
\text { presentation }\end{array}$ & $\begin{array}{l}\text { Profa Heliana Conde } \\
\text { Rodrigues, Profa Regina } \\
\text { Benevides }\end{array}$ & & & \\
\hline 01/07/94 & $\begin{array}{l}\text { Monograph } \\
\text { presentation }\end{array}$ & $\begin{array}{l}\text { Rita de Cássia Lyrio } \\
\text { Brandão }\end{array}$ & $\begin{array}{l}\text { The child, the family and systemic } \\
\text { therapy: a universe in constant } \\
\text { evolution }\end{array}$ & & \\
\hline 08/07/94 & $\begin{array}{l}\text { Thematic } \\
\text { presentation }\end{array}$ & $\begin{array}{l}\text { Profa Jane Araújo Russo, Dr } \\
\text { Erickson Furtado }\end{array}$ & $\begin{array}{l}\text { Brazil's participation in international } \\
\text { psychiatric care: Hamburg congress; } \\
\text { Scientific exchanges and the WHO } \\
\text { mental health division }\end{array}$ & & \\
\hline $15 / 07 / 94$ & $\begin{array}{l}\text { Thematic } \\
\text { presentation }\end{array}$ & $\begin{array}{l}\text { Dr José Henrique } \\
\text { Figueiredo, Dr Marco } \\
\text { Antonio Brasil, Dr Ivan } \\
\text { Figueira, Dra Heloisa } \\
\text { Ferreira }\end{array}$ & $\begin{array}{l}\text { Psychiatry and cardiology: care } \\
\text { interactions }\end{array}$ & ABP/APERJ & \\
\hline
\end{tabular}




\begin{tabular}{|c|c|c|c|c|c|}
\hline Date & Event type & Presented by & Theme & Journal/Font & Event place \\
\hline 22/07/94 & $\begin{array}{l}\text { Thematic } \\
\text { presentation }\end{array}$ & $\begin{array}{l}\text { Psic Lia Ganc and equipe } \\
\text { do setor de família/IPUB }\end{array}$ & Family in the mirror & & \\
\hline 29/07/94 & $\begin{array}{l}\text { Thematic } \\
\text { presentation }\end{array}$ & $\begin{array}{l}\text { Profa Maria da Gloria } \\
\text { Ribeiro da Silva and equipe }\end{array}$ & $\begin{array}{l}\text { Work organization and mental health } \\
\text { program }\end{array}$ & & \\
\hline 05/08/94 & $\begin{array}{l}\text { Thematic } \\
\text { presentation }\end{array}$ & $\begin{array}{l}\text { Prof Luiz Eduardo Soares, } \\
\text { Prof Jurandir Freire Costa, } \\
\text { Prof Muniz Sodré }\end{array}$ & $\begin{array}{l}\text { Ethics, Society and Mental Health; III Rio } \\
\text { de Janeiro State Psychiatry Conference }\end{array}$ & & \\
\hline $12 / 08 / 94$ & $\begin{array}{l}\text { Thematic } \\
\text { presentation }\end{array}$ & $\begin{array}{l}\text { Dr Mauricio Tostes and } \\
\text { equipe }\end{array}$ & $\begin{array}{l}\text { Mental health work in the general } \\
\text { hospital: the experience of the team at } \\
\text { the HUCFF/UFRJ medical psychology } \\
\text { and mental health service }\end{array}$ & & \\
\hline $19 / 08 / 94$ & $\begin{array}{l}\text { Thematic } \\
\text { presentation }\end{array}$ & Prof Benedetto Saraceno & $\begin{array}{l}\text { Epidemiology for Quality Assessment of } \\
\text { Mental Health Services }\end{array}$ & & \\
\hline 26/08/94 & $\begin{array}{l}\text { Thematic } \\
\text { presentation }\end{array}$ & Dr Domingos Savio & $\begin{array}{l}\text { Assistance and restructuring of mental } \\
\text { health services in Brazil }\end{array}$ & & \\
\hline 09/09/94 & $\begin{array}{l}\text { Thematic } \\
\text { presentation }\end{array}$ & $\begin{array}{l}\text { Profa Sherrine Njaine } \\
\text { Borges }\end{array}$ & $\begin{array}{l}\text { Metamorphosis of the body: a Freudian } \\
\text { pedagogy }\end{array}$ & & \\
\hline 16/09/94 & $\begin{array}{l}\text { Thematic } \\
\text { presentation }\end{array}$ & Dr Jayme Bisker & $\begin{array}{l}\text { Treatment of group patients in the } \\
\text { institution }\end{array}$ & ABP/APERJ & \\
\hline 23/09/94 & $\begin{array}{l}\text { Thematic } \\
\text { presentation }\end{array}$ & Prof Carlos Edson Duarte & Nietzsche: tragedy of madness & & \\
\hline $30 / 09 / 94$ & $\begin{array}{l}\text { Thematic } \\
\text { presentation }\end{array}$ & Prof Carlos Edson Duarte & The normality myth & & \\
\hline 07/10/94 & $\begin{array}{l}\text { Thematic } \\
\text { presentation }\end{array}$ & Dr Mauricio Tostes & $\begin{array}{l}\text { AIDS: implications for psychiatric } \\
\text { services }\end{array}$ & & \\
\hline $14 / 10 / 94$ & $\begin{array}{l}\text { Thematic } \\
\text { presentation }\end{array}$ & Prof Roberto Castel & $\begin{array}{l}\text { The crisis of the specificity of mental } \\
\text { medicine }\end{array}$ & & \\
\hline $21 / 10 / 94$ & Homage & $\begin{array}{l}\text { Profa Vera Halfoun, Prof } \\
\text { João Ferreira da Silva Filho }\end{array}$ & $\begin{array}{l}\text { Possession of the deliberative council of } \\
\text { IPUB and conference: CCS/UFRJ Project }\end{array}$ & & \\
\hline 28/10/94 & $\begin{array}{l}\text { Thematic } \\
\text { presentation }\end{array}$ & Prof João Romildo Bueno & Depression Pharmacotherapy & & \\
\hline $04 / 11 / 94$ & Homage & $\begin{array}{l}\text { Profa Maria Cristina Loyola, } \\
\text { T.O. Maria das Graças } \\
\text { Ribeiro, Psic Gina Ferreira }\end{array}$ & $\begin{array}{l}\text { Psico-Habana - } 35^{\circ} \text { anniversary of } \\
\text { Havana's Hospital in Cuba }\end{array}$ & & \\
\hline $11 / 11 / 94$ & $\begin{array}{l}\text { Thematic } \\
\text { presentation }\end{array}$ & $\begin{array}{l}\text { Clarice Moura Costa, Elieth } \\
\text { Nick }\end{array}$ & $\begin{array}{l}\text { Music therapy, language and } \\
\text { subjectivity }\end{array}$ & & \\
\hline 18/11/94 & $\begin{array}{l}\text { Thematic } \\
\text { presentation }\end{array}$ & Prof William Asmar & $\begin{array}{l}\text { Anxiety syndrome: an existential } \\
\text { approach }\end{array}$ & ABP/APERJ & \\
\hline 25/11/94 & $\begin{array}{l}\text { Thematic } \\
\text { presentation }\end{array}$ & Profa Edith Seligmann Silva & Work and Mental Health & & \\
\hline 02/12/94 & $\begin{array}{l}\text { PhD thesis } \\
\text { defense }\end{array}$ & $\begin{array}{l}\text { Dra Carla de Souza } \\
\text { Marques }\end{array}$ & Social Phobia: Comorbidity Study & & \\
\hline 09/12/94 & & $\begin{array}{l}\text { Prof João Ferreira da Silva } \\
\text { Filho and outros }\end{array}$ & Municipal Forum of mental health & & \\
\hline $16 / 12 / 94$ & $\begin{array}{l}\text { Thematic } \\
\text { presentation }\end{array}$ & $\begin{array}{l}\text { Prof Edson Saggese and } \\
\text { Equipe }\end{array}$ & $\begin{array}{l}\text { Play Project: Child } 94 \text { Award from the } \\
\text { ABRINQ Foundation for Children's } \\
\text { Rights }\end{array}$ & & \\
\hline 06/01/95 & $\begin{array}{l}\text { Thematic } \\
\text { presentation }\end{array}$ & Prof Vladimir Lazarev & $\begin{array}{l}\text { The structure of the EEG in normal } \\
\text { activity and in psychiatric patients: a } \\
\text { multidisciplinary approach }\end{array}$ & & \\
\hline 13/01/95 & $\begin{array}{l}\text { Thematic } \\
\text { presentation }\end{array}$ & Prof Paulo Mattos & $\begin{array}{l}\text { Neuropsychiatric Manifestations of } \\
\text { HIV-1 Infection }\end{array}$ & & \\
\hline 27/01/95 & $\begin{array}{l}\text { Thematic } \\
\text { presentation }\end{array}$ & Prof Jerson Laks & $\begin{array}{l}\text { Neuroscience laboratory: } \\
\text { psychogeriatrics project }\end{array}$ & & \\
\hline 10/03/95 & $\begin{array}{l}\text { Thematic } \\
\text { presentation }\end{array}$ & Prof Gillberto Velho & $\begin{array}{l}\text { Anthropology and psychiatry: Some } \\
\text { questions about Brazilian culture }\end{array}$ & & \\
\hline
\end{tabular}




\begin{tabular}{|c|c|c|c|c|c|}
\hline Date & Event type & Presented by & Theme & Journal/Font & Event place \\
\hline 17/03/95 & $\begin{array}{l}\text { Masters } \\
\text { dissertation }\end{array}$ & Dra Sandra Fortes & $\begin{array}{l}\text { Alexithymia and educational sociological } \\
\text { aspects: a study of factors linked to the } \\
\text { verbalization of emotion }\end{array}$ & & \\
\hline 24/03/95 & $\begin{array}{l}\text { Thematic } \\
\text { presentation }\end{array}$ & $\begin{array}{l}\text { Prof Jurandir Freire Costa, } \\
\text { Prof Julio Vertzman }\end{array}$ & Adolescents' discourse on sexuality & & \\
\hline $31 / 03 / 95$ & $\begin{array}{l}\text { Thematic } \\
\text { presentation }\end{array}$ & Profa Anette Leibing & The cultural construction of Psychiatry & ABP/APERJ & \\
\hline 07/04/95 & $\begin{array}{l}\text { Thematic } \\
\text { presentation }\end{array}$ & $\begin{array}{l}\text { Prof Eustachio Portella } \\
\text { Nunes Filho }\end{array}$ & Psychoanalysis ans culture & & \\
\hline 28/04/95 & $\begin{array}{l}\text { Institutional } \\
\text { Congress }\end{array}$ & & & & \\
\hline 05/05/95 & $\begin{array}{l}\text { Thematic } \\
\text { presentation }\end{array}$ & Dra Carla de Meis & $\begin{array}{l}\text { World Symposium on Ethnopsychiatry } \\
\text { - Chandighard - India }\end{array}$ & & \\
\hline $12 / 05 / 95$ & $\begin{array}{l}\text { Thematic } \\
\text { presentation }\end{array}$ & $\begin{array}{l}\text { Psic Gina Ferreira, } \\
\text { Prefeitura de Angra dso } \\
\text { Reis - RJ }\end{array}$ & $\begin{array}{l}\text { "Back home". Project to support } \\
\text { dehospitalization }\end{array}$ & & \\
\hline 19/05/95 & Homage & $\begin{array}{l}\text { Prof Eustachio Portella } \\
\text { Nunes Filho and outros }\end{array}$ & Homage to Prof Adolpho Hoirisch & & \\
\hline 26/05/95 & $\begin{array}{l}\text { Thematic } \\
\text { presentation }\end{array}$ & $\begin{array}{l}\text { Prof João Ferreira da Silva } \\
\text { Filho }\end{array}$ & $\begin{array}{l}\text { Assistance Management Council - a } \\
\text { new work proposal at IPUB }\end{array}$ & & \\
\hline 02/06/95 & Homage & Dra Ana Cristina Saad & $\begin{array}{l}\text { Council of the Association of Brazilian } \\
\text { Studies on Alcoholism and Drugs - } \\
\text { AEBAD }\end{array}$ & & \\
\hline 09/06/95 & $\begin{array}{l}\text { Thematic } \\
\text { presentation }\end{array}$ & Juiz Siro Darlan & $\begin{array}{l}\text { Justice and Mental Health: on the issue } \\
\text { of adolescent offenders }\end{array}$ & & \\
\hline 16/06/95 & $\begin{array}{l}\text { Thematic } \\
\text { presentation }\end{array}$ & Prof Roberto A Piedade & $\begin{array}{l}\text { Diagnostic resources in psychiatry - } \\
\text { SPECT and brain mapping }\end{array}$ & ABP/APERJ & \\
\hline 23/06/95 & $\begin{array}{l}\text { Thematic } \\
\text { presentation }\end{array}$ & Dr Segundo Mesa Castillo & Viral etiology of schizophrenia & Hospital Psiquiátrico de Havana & \\
\hline 30/06/95 & $\begin{array}{l}\text { Thematic } \\
\text { presentation }\end{array}$ & Profa Magaly Engel & $\begin{array}{l}\text { Insanity in the city of Rio de Janeiro - } \\
1830 / 1930\end{array}$ & & \\
\hline 05/01/96 & $\begin{array}{l}\text { Masters } \\
\text { dissertation }\end{array}$ & Katia Mecler & $\begin{array}{l}\text { Dangerousness and non-imputability: a } \\
\text { study of the factors involved in } \\
\text { determining the cessation of } \\
\text { dangerousness of the mentally ill } \\
\text { offender }\end{array}$ & & \\
\hline $12 / 01 / 96$ & $\begin{array}{l}\text { Thematic } \\
\text { presentation }\end{array}$ & Paulo Mattos & Dementia and normal aging & & \\
\hline 19/01/96 & $\begin{array}{l}\text { Thematic } \\
\text { presentation }\end{array}$ & Prof Vladimir Lazarev & $\begin{array}{l}\text { Some methodological problems of the } \\
\text { EEG investigations in Psychiatry }\end{array}$ & & \\
\hline 26/01/96 & $\begin{array}{l}\text { Masters } \\
\text { dissertation }\end{array}$ & Leila Tannous & $\begin{array}{l}\text { The clinic and diagnostic classifications } \\
\text { in psychiatry: a study of hysteria }\end{array}$ & & \\
\hline 08/03/96 & $\begin{array}{l}\text { Thematic } \\
\text { presentation }\end{array}$ & Prof Luis Pinguelli Rosa & $\begin{array}{l}\text { Inaugural class - The Brazilian university } \\
\text { and the production of science and } \\
\text { technology }\end{array}$ & & \\
\hline $15 / 03 / 96$ & Homage & $\begin{array}{l}\text { Domingos Saio N Alves, } \\
\text { Alfredo Schnechtman, Hugo } \\
\text { Marques Fagundes, Maria } \\
\text { Tavares Cavalcanti }\end{array}$ & $\begin{array}{l}\text { Perspectives in Psychiatric Care in } \\
\text { Transformation; Release of IPUB's } \\
\text { notebooks n³ }\end{array}$ & & \\
\hline 22/03/96 & $\begin{array}{l}\text { Thematic } \\
\text { presentation }\end{array}$ & $\begin{array}{l}\text { Jerson Laks, Eliasz } \\
\text { Engelhardt }\end{array}$ & Emotions neuropsychology & & \\
\hline 29/03/96 & $\begin{array}{l}\text { Masters } \\
\text { dissertation }\end{array}$ & $\begin{array}{l}\text { Roberto Santoro P de } \\
\text { Carvalho Almeida }\end{array}$ & $\begin{array}{l}\text { The organization of a mental health } \\
\text { service in a Brazilian public pediatric } \\
\text { hospital: a case study }\end{array}$ & & \\
\hline $12 / 04 / 96$ & $\begin{array}{l}\text { Thematic } \\
\text { presentation }\end{array}$ & Annette Leibing & $\begin{array}{l}\text { Writing in the Margins: One Month at } \\
\text { Harvard }\end{array}$ & & \\
\hline
\end{tabular}




\begin{tabular}{|c|c|c|c|c|}
\hline Date & Event type & Presented by & Journal/Font & Event place \\
\hline 19/04/96 & $\begin{array}{l}\text { Thematic } \\
\text { presentation }\end{array}$ & $\begin{array}{l}\text { Talvane Moraes, Miguel } \\
\text { Chalub, Jorge Adelino, } \\
\text { Fatima Vasconcellos }\end{array}$ & Ethics in psychiatry & \\
\hline 26/04/96 & $\begin{array}{l}\text { Thematic } \\
\text { presentation }\end{array}$ & $\begin{array}{l}\text { Leticia L Vermelho, Regina } \\
\text { Helena S Barbosa }\end{array}$ & Women and the epidemiology of AIDS & \\
\hline 03/05/96 & $\begin{array}{l}\text { Institutional } \\
\text { Congress }\end{array}$ & $\begin{array}{l}\text { Jane Russo, Ana Cristina } \\
\text { Figueiredo and outros }\end{array}$ & $\begin{array}{l}\text { XIX Institutional Congress of } \\
\text { Specialization Courses }\end{array}$ & \\
\hline $10 / 05 / 96$ & $\begin{array}{l}\text { Masters } \\
\text { dissertation }\end{array}$ & Carla Meis & $\begin{array}{l}\text { Prostitution, a profession? Liminality, } \\
\text { death and emptiness in the trajectory of } \\
\text { acquiring an identity as a prostitute }\end{array}$ & \\
\hline $17 / 05 / 96$ & $\begin{array}{l}\text { Thematic } \\
\text { presentation }\end{array}$ & Maria Tavares Cavalcanti & $\begin{array}{l}\text { The "IPUB case": in search of total } \\
\text { quality }\end{array}$ & \\
\hline $24 / 05 / 96$ & $\begin{array}{l}\text { PhD thesis } \\
\text { defense }\end{array}$ & Marcio Amaral & $\begin{array}{l}\text { Are the moral values of persistent } \\
\text { criminals different from those of general } \\
\text { citizens? }\end{array}$ & \\
\hline $30 / 05 / 96$ & $\begin{array}{l}\text { Thematic } \\
\text { presentation }\end{array}$ & $\begin{array}{l}\text { Prof John Mack from } \\
\text { Harvard University }\end{array}$ & $\begin{array}{l}\text { The phenomenon of alien abductions } \\
\text { - a matter of psychiatry? - Special } \\
\text { Conference }\end{array}$ & \\
\hline $31 / 05 / 96$ & $\begin{array}{l}\text { Thematic } \\
\text { presentation }\end{array}$ & Carmen Tourinho & The case of the IPUB infirmary & \\
\hline 14/06/96 & $\begin{array}{l}\text { Thematic } \\
\text { presentation }\end{array}$ & Prof Miguel Chalub & $\begin{array}{l}\text { A case of forensic psychiatry l: The } \\
\text { copacabana hitman }\end{array}$ & \\
\hline 21/06/96 & $\begin{array}{l}\text { Thematic } \\
\text { presentation }\end{array}$ & $\begin{array}{l}\text { Prof João Ferreira da Silva } \\
\text { Filho }\end{array}$ & $\begin{array}{l}\text { The University Health Institutions and } \\
\text { the UFRJ }\end{array}$ & \\
\hline 28/06/96 & $\begin{array}{l}\text { Thematic } \\
\text { presentation }\end{array}$ & Marco Antonio Brasil & Patients with diffuse complaints & \\
\hline 05/07/96 & $\begin{array}{l}\text { Thematic } \\
\text { presentation }\end{array}$ & Lucia Duarte & $\begin{array}{l}\text { The formation of rational thought in } \\
\text { archaic greece: a Jungian view }\end{array}$ & \\
\hline 08/07/96 & $\begin{array}{l}\text { Thematic } \\
\text { presentation }\end{array}$ & $\begin{array}{l}\text { Denis Turk from Pittsburgh } \\
\text { University }\end{array}$ & $\begin{array}{l}\text { First mental health encounter in a pain } \\
\text { clinic }\end{array}$ & \\
\hline 12/07/96 & Homage & $\begin{array}{l}\text { Prof Eustachio Portella } \\
\text { Nunes Filho, Prof João } \\
\text { Ferreira Filho, Fatima } \\
\text { Vasconcellos }\end{array}$ & Homage to Prof José Candido & \\
\hline 19/07/96 & $\begin{array}{l}\text { Thematic } \\
\text { presentation }\end{array}$ & Sergio Levcovitz and cols & The IPUB outpatient reception group & \\
\hline 26/07/96 & $\begin{array}{l}\text { Thematic } \\
\text { presentation }\end{array}$ & $\begin{array}{l}\text { Maria Tavares Cavalcanti, } \\
\text { Antonio Egidio Nardi }\end{array}$ & $\begin{array}{l}\text { Conference reports: } V \text { international day } \\
\text { of the mental health aid union in } \\
\text { Bordeaux, France; Congress of the } \\
\text { International College of } \\
\text { Neuropsychopharmacology - Sydney, } \\
\text { Australia }\end{array}$ & \\
\hline 02/08/96 & Homage & $\begin{array}{l}\text { Prof Eustachio Portella } \\
\text { Nunes Filho }\end{array}$ & $\begin{array}{l}\text { 58th Anniversary of IPUB - Psychiatry } \\
\text { and mental health }\end{array}$ & \\
\hline 10/08/96 & $\begin{array}{l}\text { Thematic } \\
\text { presentation }\end{array}$ & $\begin{array}{l}\text { Alfredo Schechtman, Edson } \\
\text { Saggese, João ferreira Filho } \\
\text { and outros }\end{array}$ & $\begin{array}{l}\text { V Rio de Janeiro State Psychiatry } \\
\text { Conference: Mental Health in Childhood } \\
\text { and Adolescence }\end{array}$ & \\
\hline $12 / 08 / 96$ & $\begin{array}{l}\text { Thematic } \\
\text { presentation }\end{array}$ & $\begin{array}{l}\text { Daniel Sampaio from Lisbon } \\
\text { University }\end{array}$ & $\begin{array}{l}\text { Special Conference - Family Therapy } \\
\text { and the Troubled Adolescent }\end{array}$ & \\
\hline 16/08/96 & $\begin{array}{l}\text { Thematic } \\
\text { presentation }\end{array}$ & Stevens Kastrup Rehens & $\begin{array}{l}\text { Study of Apoptosis in the Central } \\
\text { Nervous System }\end{array}$ & \\
\hline 23/08/96 & $\begin{array}{l}\text { Thematic } \\
\text { presentation }\end{array}$ & Carlos Edson Duarte & Research in Psychoanalysis & \\
\hline $30 / 08 / 96$ & $\begin{array}{l}\text { Thematic } \\
\text { presentation }\end{array}$ & $\begin{array}{l}\text { Michel Demangeat from } \\
\text { Bordeaux University }\end{array}$ & $\begin{array}{l}\text { Difficulties in Historicization in the } \\
\text { Psychotherapy of Psychotic Subjects }\end{array}$ & \\
\hline
\end{tabular}




\begin{tabular}{|c|c|c|c|c|c|}
\hline Date & Event type & Presented by & Theme & Journal/Font & Event place \\
\hline 06/09/96 & $\begin{array}{l}\text { Thematic } \\
\text { presentation }\end{array}$ & $\begin{array}{l}\text { Mauricio Tostes, Suely } \\
\text { Broxado }\end{array}$ & $\begin{array}{l}\text { AIDS and sexuality: some relevant } \\
\text { aspects of the 11th international } \\
\text { conference in Vancouver, Canada }\end{array}$ & & \\
\hline 13/09/96 & $\begin{array}{l}\text { Thematic } \\
\text { presentation }\end{array}$ & Miguel Chalub & $\begin{array}{l}\text { A Case of Forensic Psychiatry II: Raptus } \\
\text { Melancholicus Murder of Own Children }\end{array}$ & & \\
\hline 20/09/96 & $\begin{array}{l}\text { Thematic } \\
\text { presentation }\end{array}$ & José Angelo Pappi & $\begin{array}{l}\text { Neuropsychiatric Manifestations of } \\
\text { Systemic Lupus Erythrematosus: } \\
\text { Experience of the University Hospital }\end{array}$ & & \\
\hline 27/09/96 & $\begin{array}{l}\text { Thematic } \\
\text { presentation }\end{array}$ & $\begin{array}{l}\text { Antonio Quinet from Paris } \\
\text { VII University }\end{array}$ & The Bishop case & Universidade de Paris VII & \\
\hline $11 / 10 / 96$ & $\begin{array}{l}\text { Thematic } \\
\text { presentation }\end{array}$ & $\begin{array}{l}\text { José Alberto Zusman, Maria } \\
\text { Tavares Cavalcanti, Nelson } \\
\text { Goldstein, Abmael de Souza } \\
\text { Alves, Sergio Levcovitz }\end{array}$ & $\begin{array}{l}\text { Daily Care Center of the Institute of } \\
\text { Psychiatry - IPUB }\end{array}$ & & \\
\hline 18/10/96 & $\begin{array}{l}\text { Thematic } \\
\text { presentation }\end{array}$ & Vera Pollo & The clinical structure of Hysteria & & \\
\hline 25/10/96 & Homage & $\begin{array}{l}\text { Maria Paula Cerqueira and } \\
\text { cols }\end{array}$ & $\begin{array}{l}\text { Public health policies in the municipality } \\
\text { of Angra dos Reis - RJ; Release of } \\
\text { IPUB's notebooks n }{ }^{\circ} 4\end{array}$ & & \\
\hline 01/11/96 & $\begin{array}{l}\text { Thematic } \\
\text { presentation }\end{array}$ & Paulo Novaes & Perspectives for the elderly & & \\
\hline 08/11/96 & $\begin{array}{l}\text { Thematic } \\
\text { presentation }\end{array}$ & Jens Schneider & $\begin{array}{l}\text { Identity, discourse and cultural } \\
\text { citizenship: on the construction of } \\
\text { "Social belonging" }\end{array}$ & & \\
\hline $\begin{array}{l}20 \mathrm{a} \\
23 / 11 / 96\end{array}$ & & $\begin{array}{l}\text { Jurandir Freire Costa, } \\
\text { Genevieve Denys, Jorge } \\
\text { Alberto Costa and Silva }\end{array}$ & $\begin{array}{l}\text { 1st mental health congress of the state } \\
\text { of Rio de Janeiro: paradigms of } \\
\text { psychosocial care }\end{array}$ & & \\
\hline 29/11/96 & $\begin{array}{l}\text { Thematic } \\
\text { presentation }\end{array}$ & Elisaneth Seporiti & $\begin{array}{l}\text { A theory of interpretation in the face of } \\
\text { symptom impasses }\end{array}$ & & \\
\hline 06/12/96 & $\begin{array}{l}\text { Thematic } \\
\text { presentation }\end{array}$ & $\begin{array}{l}\text { Sergio Carrara; Patrick } \\
\text { Larvie }\end{array}$ & $\begin{array}{l}\text { Tribute to Venus: the fight against } \\
\text { syphilis in Brazil; AIDS and the } \\
\text { behavioral sciences }\end{array}$ & & \\
\hline $13 / 12 / 96$ & $\begin{array}{l}\text { Thematic } \\
\text { presentation }\end{array}$ & $\begin{array}{l}\text { Shulamit Ramon, from } \\
\text { London University }\end{array}$ & $\begin{array}{l}\text { Closing psychiatric hospitals and } \\
\text { resettlement of patients in the } \\
\text { community: the English experience }\end{array}$ & & \\
\hline 20/12/96 & $\begin{array}{l}\text { Thematic } \\
\text { presentation }\end{array}$ & $\begin{array}{l}\text { José Maria Alberdi, from } \\
\text { Rosário Uiversity }\end{array}$ & Psychiatric Reform in Argentina & & \\
\hline $17 / 01 / 97$ & $\begin{array}{l}\text { Thematic } \\
\text { presentation }\end{array}$ & Maria Lucia Quinta & $\begin{array}{l}\text { The place of occupational therapy in } \\
\text { mental health }\end{array}$ & & \\
\hline 24/01/97 & $\begin{array}{l}\text { PhD thesis } \\
\text { defense }\end{array}$ & José Helio Ribeiro de Melo & Schizophrenia: Citizen Overload & & \\
\hline $31 / 01 / 97$ & $\begin{array}{l}\text { Thematic } \\
\text { presentation }\end{array}$ & Miguel Chalub & $\begin{array}{l}\text { Passionate fantasy murder: forensic } \\
\text { psychiatric aspects }\end{array}$ & & \\
\hline 07/02/97 & $\begin{array}{l}\text { Masters } \\
\text { dissertation }\end{array}$ & Graça Regina Lima Cadilhe & $\begin{array}{l}\text { Quality assessment of mental health } \\
\text { services in the Brazilian municipality }\end{array}$ & & \\
\hline 21/02/97 & $\begin{array}{l}\text { Thematic } \\
\text { presentation }\end{array}$ & $\begin{array}{l}\text { Prof João Ferreira da Silva } \\
\text { Filho }\end{array}$ & Assistance at the Institute of Psychiatry & & \\
\hline 28/02/97 & $\begin{array}{l}\text { Masters } \\
\text { dissertation }\end{array}$ & Nelson Goldstein & $\begin{array}{l}\text { Between the body and the soul on the } \\
\text { formation of symptoms in schizophrenic } \\
\text { psychoses }\end{array}$ & & \\
\hline 07/03/97 & $\begin{array}{l}\text { Thematic } \\
\text { presentation }\end{array}$ & Prof Leopoldo de Meis & Opening class - Science in Brazil & & \\
\hline $14 / 03 / 97$ & $\begin{array}{l}\text { Thematic } \\
\text { presentation }\end{array}$ & Max de Carvalho & $\begin{array}{l}\text { Neuropathological and clinical } \\
\text { correlates of depression in stroke } \\
\text { patients }\end{array}$ & & \\
\hline $21 / 03 / 97$ & $\begin{array}{l}\text { Thematic } \\
\text { presentation }\end{array}$ & Renato Neves & $\begin{array}{l}\text { Seniors and mental health - A critical } \\
\text { approach }\end{array}$ & & \\
\hline
\end{tabular}




\begin{tabular}{|c|c|c|c|c|c|}
\hline Date & Event type & Presented by & Theme & Journal/Font & Event place \\
\hline 04/04/97 & $\begin{array}{l}\text { PhD thesis } \\
\text { defense }\end{array}$ & Octavio Domont Serpa Jr & $\begin{array}{l}\text { Illness in Nature: A Critical Study on } \\
\text { Reductionism and Biological } \\
\text { Determinism in Psychiatry }\end{array}$ & & \\
\hline $11 / 04 / 97$ & $\begin{array}{l}\text { Thematic } \\
\text { presentation }\end{array}$ & Ivan Figueira & Anxiety and Depression Program & & \\
\hline $18 / 04 / 97$ & $\begin{array}{l}\text { Institutional } \\
\text { Congress }\end{array}$ & $\begin{array}{l}\text { Adriana Costa Machado, } \\
\text { Odacy Soares Cabeleira }\end{array}$ & $\begin{array}{l}\text { XX Institutional Congress of } \\
\text { specialization courses - An outside } \\
\text { view - a study on the dynamics of day } \\
\text { hospitals through former users }\end{array}$ & & \\
\hline 25/04/97 & $\begin{array}{l}\text { Thematic } \\
\text { presentation }\end{array}$ & $\begin{array}{l}\text { Bianca Telles Ribeiro, } \\
\text { Mariluci Novaes, Maria } \\
\text { Tavares Cavalcanti, João } \\
\text { Ferreira Filho }\end{array}$ & $\begin{array}{l}\text { Mosaic discourse: contributions from } \\
\text { linguistics and semiology to the field of } \\
\text { mental health }\end{array}$ & & \\
\hline 09/05/97 & $\begin{array}{l}\text { Masters } \\
\text { dissertation }\end{array}$ & Ana Cristina Ebrehz da Cruz & The malignant neuroleptic syndrome & & \\
\hline $16 / 05 / 97$ & $\begin{array}{l}\mathrm{PhD} \text { thesis } \\
\text { defense }\end{array}$ & Julio Sérgi Verztman & $\begin{array}{l}\text { Liberalism and cruelty: a study on the } \\
\text { notion of sexual preference }\end{array}$ & & \\
\hline 23/05/97 & Homage & $\begin{array}{l}\text { Sandra Fortes, Sergio } \\
\text { Zaidaft, José Henrique } \\
\text { Figueiredo, Leticia } \\
\text { Furlamento }\end{array}$ & $\begin{array}{l}\text { Liberalism and cruelty: a study on the } \\
\text { notion of sexual preference; Release of } \\
\text { IPUB's notebooks n'6 }\end{array}$ & & \\
\hline 06/06/97 & $\begin{array}{l}\text { Thematic } \\
\text { presentation }\end{array}$ & Benilton Bezerra Jr & $\begin{array}{l}\text { Theories of the mind-body relationship: } \\
\text { its implications for psychiatric reform in } \\
\text { Brazil }\end{array}$ & & \\
\hline 13/06/97 & $\begin{array}{l}\text { PhD thesis } \\
\text { defense }\end{array}$ & $\begin{array}{l}\text { José Carlos Borges } \\
\text { Appolinário }\end{array}$ & $\begin{array}{l}\text { Menopause: Assessment of mood } \\
\text { symptoms in women with menopause } \\
\text { who seek medical care }\end{array}$ & & \\
\hline 20/06/97 & & $\begin{array}{l}\text { João Ferreira da Silva Filho, } \\
\text { Cristina Loyola de Miranda } \\
\text { and outros }\end{array}$ & $\begin{array}{l}\text { I municipal meeting of daily care } \\
\text { services in the city of Rio de Janeiro } \\
\text { and II meeting of daily care services in } \\
\text { the state of Rio de Janeiro; The } \\
\text { challenges of building daily care } \\
\text { services in the city of Rio de Janeiro }\end{array}$ & & \\
\hline 27/06/97 & $\begin{array}{l}\text { Thematic } \\
\text { presentation }\end{array}$ & $\begin{array}{l}\text { Celine Mercier, Paul } \\
\text { Laporte, Christian Dogenais, } \\
\text { from McGill University and } \\
\text { Le cirque du soleil }\end{array}$ & $\begin{array}{l}\text { Circus workshop for children at risk: the } \\
\text { Rio de Janeiro project }\end{array}$ & & \\
\hline 04/07/97 & $\begin{array}{l}\text { Thematic } \\
\text { presentation }\end{array}$ & $\begin{array}{l}\text { Marta Rezende Cardoso, } \\
\text { from Paris VII University }\end{array}$ & The "strange" violence of the superego & & \\
\hline $11 / 07 / 97$ & $\begin{array}{l}\text { Thematic } \\
\text { presentation }\end{array}$ & $\begin{array}{l}\text { Silvia Jardim, Magda } \\
\text { Vaissman, José Mouro Brás } \\
\text { Lima }\end{array}$ & Worker support program & & \\
\hline 18/07/97 & Homage & $\begin{array}{l}\text { Eduardo Mourão } \\
\text { Vasconcellos and outros }\end{array}$ & $\begin{array}{l}\text { Mental health and deinstitutionalization } \\
\text { - reinventing services; Release of } \\
\text { IPUB's notebooks n }{ }^{0} 7\end{array}$ & Lançamento do cadernos do IPUB n7 & \\
\hline 25/07/97 & $\begin{array}{l}\text { Thematic } \\
\text { presentation }\end{array}$ & Sergio Levcovitz & $\begin{array}{l}\text { Don quixote and death: on the } \\
\text { dissolution of delusion }\end{array}$ & & \\
\hline 01/08/97 & $\begin{array}{l}\text { Thematic } \\
\text { presentation }\end{array}$ & Maria Tereza Vieira & The importance of art in mental health & & \\
\hline 08/08/97 & Homage & João Ferreira da Silva Filho & $\begin{array}{l}\text { 59th Anniversary of IPUB - The Work of } \\
\text { IPUB }\end{array}$ & & \\
\hline $15 / 08 / 97$ & $\begin{array}{l}\text { Thematic } \\
\text { presentation }\end{array}$ & Marcio Amaral & $\begin{array}{l}\text { Classifications in psychiatry: where it } \\
\text { sees and where it tends }\end{array}$ & & \\
\hline 22/08/97 & $\begin{array}{l}\text { Thematic } \\
\text { presentation }\end{array}$ & Marcus André Vieira & The mania: a Freudian reading & & \\
\hline $\begin{array}{l}28- \\
30 / 08 / 97\end{array}$ & & $\begin{array}{l}\text { João Ferreira da Silva Filho, } \\
\text { Leonardo Boff and outros }\end{array}$ & $\begin{array}{l}\text { VI Journey of Psychiatry in the State of } \\
\text { Rio de Janeiro: interdisciplinarity in } \\
\text { psychiatry }\end{array}$ & & \\
\hline
\end{tabular}




\begin{tabular}{|c|c|c|c|c|}
\hline Date & Event type & Presented by & Journal/Font & Event place \\
\hline 05/09/97 & $\begin{array}{l}\text { Thematic } \\
\text { presentation }\end{array}$ & $\begin{array}{l}\text { Eliasz Engelhardt, Jerson } \\
\text { Laks }\end{array}$ & $\begin{array}{l}\text { Cognitive Tracking in Elderly } 65 \text { to } 108 \\
\text { years old in RJ }\end{array}$ & \\
\hline $12 / 09 / 97$ & $\begin{array}{l}\text { Thematic } \\
\text { presentation }\end{array}$ & $\begin{array}{l}\text { Christopher Bolles, from } \\
\text { England }\end{array}$ & Mind against self & \\
\hline $19 / 09 / 97$ & $\begin{array}{l}\text { Thematic } \\
\text { presentation }\end{array}$ & $\begin{array}{l}\text { Cristina Ma Loyola Miranda, } \\
\text { Manoel Olavo Teixeira }\end{array}$ & Hospital work at IPUB & \\
\hline 26/09/97 & $\begin{array}{l}\text { PhD thesis } \\
\text { defense }\end{array}$ & Elie Cheniaux Junior & $\begin{array}{l}\text { The inclusion of premenstrual syndrome } \\
\text { among mental disorders }\end{array}$ & \\
\hline 03/10/97 & & & IPUB's Assistance meeting & \\
\hline $10 / 10 / 97$ & $\begin{array}{l}\text { PhD thesis } \\
\text { defense }\end{array}$ & Lidia Soares Cardoso & Banking work and professional identity & \\
\hline $17 / 10 / 97$ & $\begin{array}{l}\text { Thematic } \\
\text { presentation }\end{array}$ & $\begin{array}{l}\text { Carmem Tourinho, Denise } \\
\text { Correa, Mariangela Aleixo }\end{array}$ & $\begin{array}{l}\text { Mental Health Congress in Havana/ } \\
\text { Cuba }\end{array}$ & \\
\hline 20/10/97 & $\begin{array}{l}\text { Thematic } \\
\text { presentation }\end{array}$ & Antonio Pacheco Palha & $\begin{array}{l}\text { Special conference - Somatization } \\
\text { mechanisms in psychiatric clinic and } \\
\text { consultation }\end{array}$ & \\
\hline 24/10/97 & $\begin{array}{l}\text { Thematic } \\
\text { presentation }\end{array}$ & $\begin{array}{l}\text { Marco Antonio Brasil, João } \\
\text { Ferreira da Silva Filho, Hugo } \\
\text { Fagundes, Sergio Zaidahff }\end{array}$ & Psychiatry in a General Hospital & \\
\hline $31 / 10 / 97$ & $\begin{array}{l}\text { Thematic } \\
\text { presentation }\end{array}$ & Luiz Fernando D Duarte & $\begin{array}{l}\text { Modern western culture and an } \\
\text { anthropology of the person }\end{array}$ & \\
\hline 07/11/97 & $\begin{array}{l}\text { Thematic } \\
\text { presentation }\end{array}$ & $\begin{array}{l}\text { Nelson Goldstein, José } \\
\text { Alberto Zusman }\end{array}$ & $\begin{array}{l}\text { Teaching and Research on new IPUB } \\
\text { assistive devices }\end{array}$ & \\
\hline $14 / 11 / 97$ & Homage & João Romildo Bueno & $\begin{array}{l}25 \text { years of postgraduate studies in } \\
\text { psychiatry in Brazil }\end{array}$ & \\
\hline 21/11/97 & Homage & $\begin{array}{l}\text { Sergio Levcovitz, Katia } \\
\text { Mecler, Alexandre Valença, } \\
\text { Katia Petribu, José Manoel } \\
\text { Bertolote, Talvane de } \\
\text { Moraes, Rogerio W Aguiar }\end{array}$ & $\begin{array}{l}\text { Ceremony for the delivery of the Luiz } \\
\text { Cerqueira, Rui de Pinho and ABP } \\
\text { awards }\end{array}$ & \\
\hline 28/11/97 & $\begin{array}{l}\text { Thematic } \\
\text { presentation }\end{array}$ & $\begin{array}{l}\text { Jane Russo, Lygia Costa } \\
\text { Leite, Annette Leibing }\end{array}$ & Culture and Mental health & \\
\hline 05/12/97 & $\begin{array}{l}\text { Thematic } \\
\text { presentation }\end{array}$ & $\begin{array}{l}\text { Prof Eustachio Portella } \\
\text { Nunes Filho }\end{array}$ & $\begin{array}{l}\text { Is Freud's work just Shakespeare in } \\
\text { prose? }\end{array}$ & \\
\hline $12 / 12 / 97$ & $\begin{array}{l}\text { PhD thesis } \\
\text { defense }\end{array}$ & Maria Tavares Cavalcanti & $\begin{array}{l}\text { The weave of the loom: about treating it } \\
\text { in psychiatry }\end{array}$ & \\
\hline $19 / 12 / 97$ & $\begin{array}{l}\text { Thematic } \\
\text { presentation }\end{array}$ & $\begin{array}{l}\text { Annette Leibing, Jerson } \\
\text { Laks, Jacov Guteman, Lucia } \\
\text { Leme }\end{array}$ & & \\
\hline 08/01/99 & $\begin{array}{l}\text { Thematic } \\
\text { presentation }\end{array}$ & Jeremias Ferraz Lima & $\begin{array}{l}\text { The paradigms of psychoanalysis and } \\
\text { the Research Problem }\end{array}$ & \\
\hline $15 / 01 / 99$ & $\begin{array}{l}\text { Thematic } \\
\text { presentation }\end{array}$ & Eduardo Perillo & $\begin{array}{l}\text { Organized interests in health and } \\
\text { resistance to change }\end{array}$ & \\
\hline 22/01/99 & $\begin{array}{l}\text { PhD thesis } \\
\text { defense }\end{array}$ & Luis Henrique Borges & $\begin{array}{l}\text { Sociabilidade, sofrimento psíquico and } \\
\text { lesões por esforço repetitivos em } \\
\text { processos de trabalhos repetitivos: } \\
\text { Estudo de caixas bancáriasSociability, } \\
\text { psychological distress and repetitive } \\
\text { stress injuries in repetitive work } \\
\text { processes: Study of bank tellers }\end{array}$ & \\
\hline 29/01/99 & $\begin{array}{l}\text { Thematic } \\
\text { presentation }\end{array}$ & Cleto Brasileiro Pontes & Human Insights and Mimetism & \\
\hline 05/03/99 & $\begin{array}{l}\text { Thematic } \\
\text { presentation }\end{array}$ & Emanuel Carneiro Leão & $\begin{array}{l}\text { Thought in Philosophy and the } \\
\text { University }\end{array}$ & \\
\hline
\end{tabular}




\begin{tabular}{|c|c|c|c|c|}
\hline Date & Event type & Presented by & Journal/Font & Event place \\
\hline 12/03/99 & $\begin{array}{l}\text { PhD thesis } \\
\text { defense }\end{array}$ & $\begin{array}{l}\text { Manoel Olavo Loureiro } \\
\text { Teixeira }\end{array}$ & $\begin{array}{l}\text { God and science in the land of the sun: } \\
\text { Pedro Il's hospice and the constitution } \\
\text { of mental medicine in Brazil }\end{array}$ & \\
\hline 19/03/99 & $\begin{array}{l}\text { Thematic } \\
\text { presentation }\end{array}$ & $\begin{array}{l}\text { Sibylle Benninghoff-Lühl, } \\
\text { from Humboldt University }\end{array}$ & $\begin{array}{l}\text { Psychoanalysis and literature - } \\
\text { Sigmund Freud's relation to Johann } \\
\text { Wolfgang Goethe }\end{array}$ & \\
\hline 26/03/99 & $\begin{array}{l}\text { Thematic } \\
\text { presentation }\end{array}$ & $\begin{array}{l}\text { José Carlos Borges } \\
\text { Appolinário }\end{array}$ & $\begin{array}{l}\text { New Pharmacological Agents in the } \\
\text { Treatment of Obesity: Psychiatric } \\
\text { Implications }\end{array}$ & \\
\hline 09/04/99 & $\begin{array}{l}\text { Thematic } \\
\text { presentation }\end{array}$ & $\begin{array}{l}\text { Celine Mercier, Pedro } \\
\text { Gabriel Delgado, from } \\
\text { McGill University }\end{array}$ & $\begin{array}{l}\text { Public Policies and Quality of Life in } \\
\text { Mental Health; Release of IPUB's } \\
\text { notebooks } n^{0} 14\end{array}$ & \\
\hline 16/04/99 & $\begin{array}{l}\text { Thematic } \\
\text { presentation }\end{array}$ & $\begin{array}{l}\text { Rolf Wirsing, from Görlitz } \\
\text { University and Leipizig } \\
\text { University }\end{array}$ & $\begin{array}{l}\text { The body as an object - Antopological } \\
\text { perspectives }\end{array}$ & \\
\hline 23/04/99 & $\begin{array}{l}\text { Thematic } \\
\text { presentation }\end{array}$ & Clara Helena Portella Nunes & Psychotherapy and Psychoanalysis & \\
\hline 30/04/99 & $\begin{array}{l}\text { Congress of } \\
\text { IPUB's } \\
\text { specialization } \\
\text { courses }\end{array}$ & & & \\
\hline 07/05/99 & $\begin{array}{l}\text { Thematic } \\
\text { presentation }\end{array}$ & $\begin{array}{l}\text { Francine Saillant, from } \\
\text { Laval University }\end{array}$ & $\begin{array}{l}\text { Health and responsibility - a } \\
\text { comparative study between populations } \\
\text { of quebec and amazons }\end{array}$ & \\
\hline 14/05/99 & $\begin{array}{l}\text { Thematic } \\
\text { presentation }\end{array}$ & $\begin{array}{l}\text { Silvia Rodrigues Jardim, } \\
\text { Carlos Lessa, Jane Souto, } \\
\text { Lygia Costa Leite }\end{array}$ & Work and the adolescent & \\
\hline 21/05/99 & $\begin{array}{l}\text { Thematic } \\
\text { presentation }\end{array}$ & $\begin{array}{l}\text { Fernando Ramos, Frederico } \\
\text { Vasconcelos, João Carlos } \\
\text { Dias, Mario Biscaia }\end{array}$ & $\begin{array}{l}\text { Update on alcoholism - Diagnosis, } \\
\text { treatment and standardization }\end{array}$ & \\
\hline 28/05/99 & $\begin{array}{l}\text { Thematic } \\
\text { presentation }\end{array}$ & Lia Ganc & $\begin{array}{l}\text { Voice and silence: communication in } \\
\text { the families of survivors in extreme } \\
\text { situations }\end{array}$ & \\
\hline 11/06/99 & $\begin{array}{l}\text { Thematic } \\
\text { presentation }\end{array}$ & $\begin{array}{l}\text { Patrick Larvie, from } \\
\text { Chicago University }\end{array}$ & $\begin{array}{l}\text { Psychology as a technique of political } \\
\text { mediation }\end{array}$ & \\
\hline 18/06/99 & & Antonio Egidio Nardi & $\begin{array}{l}\text { Reports from the Congress of the } \\
\text { American Psychiatric Association }\end{array}$ & \\
\hline 25/06/99 & $\begin{array}{l}\text { Thematic } \\
\text { presentation }\end{array}$ & Antonio Quinet & $\begin{array}{l}\text { The presentation of patients in } \\
\text { psychoanalytical orientation }\end{array}$ & \\
\hline 02/07/99 & $\begin{array}{l}\text { Thematic } \\
\text { presentation }\end{array}$ & Miguel Chalub & $\begin{array}{l}\text { Crime and madness: considerations on } \\
\text { a case of homicide in a delusional } \\
\text { hallucinatory episode. Criminal liability } \\
\text { in psychoses }\end{array}$ & \\
\hline 09/07/99 & $\begin{array}{l}\text { Thematic } \\
\text { presentation }\end{array}$ & Marcio Versiani & Social Functioning and Depression & \\
\hline 16/07/99 & $\begin{array}{l}\text { Thematic } \\
\text { presentation }\end{array}$ & Elie Cheniaux & $\begin{array}{l}\text { Menstruation: a psychoanalytic } \\
\text { approach }\end{array}$ & \\
\hline 23/07/99 & $\begin{array}{l}\text { PhD thesis } \\
\text { defense }\end{array}$ & Carla de Meis & $\begin{array}{l}\text { Jardim de Sombras: AIDS rescue and } \\
\text { prevention movements among } \\
\text { prostitutes and prostitutes in Rio de } \\
\text { Janeiro }\end{array}$ & \\
\hline 30/07/99 & $\begin{array}{l}\text { SOSINTRA } \\
\text { Simposium }\end{array}$ & & & \\
\hline 06/08/99 & $\begin{array}{l}\text { Thematic } \\
\text { presentation }\end{array}$ & $\begin{array}{l}\text { Marcia Rozenthal, Nelson } \\
\text { Maculam }\end{array}$ & $\begin{array}{l}\text { Artificial neural networks for tracking } \\
\text { cognitive patterns in schizophrenia }\end{array}$ & \\
\hline 13/08/99 & $\begin{array}{l}\text { Thematic } \\
\text { presentation }\end{array}$ & $\begin{array}{l}\text { Theodor Lowenkron, Joel } \\
\text { Birman }\end{array}$ & Is brief psychoanalysis possible? & \\
\hline
\end{tabular}




\begin{tabular}{|c|c|c|c|c|}
\hline Date & Event type & Presented by & Journal/Font & Event place \\
\hline 28/08/99 & $\begin{array}{l}\text { Thematic } \\
\text { presentation }\end{array}$ & $\begin{array}{l}\text { Marina Bandeira, Madalena } \\
\text { Libério }\end{array}$ & $\begin{array}{l}\text { Deinstitutionalization: Importance of } \\
\text { community mental health infrastructure }\end{array}$ & \\
\hline 27/08/99 & $\begin{array}{l}\text { Thematic } \\
\text { presentation }\end{array}$ & Daniel Groisman & $\begin{array}{l}\text { The childhood of the asylum: } \\
\text { institutionalization from the historical } \\
\text { point of view }\end{array}$ & \\
\hline 03/09/99 & Homage & João Ferreira da Silva Filho & $\begin{array}{l}61 \text { years of IPUB - Art exhibition by an } \\
\text { ipub patient, video and release of ipub's } \\
\text { 15th notebook }\end{array}$ & \\
\hline 10/09/99 & $\begin{array}{l}\text { Thematic } \\
\text { presentation }\end{array}$ & Miguel Chalub, Katia Mecler & $\begin{array}{l}\text { A Case of Forensic Psychiatry: The } \\
\text { Doctor Killing Doctors }\end{array}$ & \\
\hline 17/09/99 & $\begin{array}{l}\text { Thematic } \\
\text { presentation }\end{array}$ & $\begin{array}{l}\text { João Ferreira da Silva Filho, } \\
\text { Edson Saggese, Lygia Costa } \\
\text { Leite, Ana Maria Pitta }\end{array}$ & $\begin{array}{l}\text { Child and Youth Psychosocial Care } \\
\text { Center }\end{array}$ & \\
\hline 24/09/99 & $\begin{array}{l}\text { Thematic } \\
\text { presentation }\end{array}$ & Maria Clara Queiroz Correa & $\begin{array}{l}\text { The experience of excess - for a review } \\
\text { of the madness of artists }\end{array}$ & \\
\hline 01/10/99 & $\begin{array}{l}\text { Thematic } \\
\text { presentation }\end{array}$ & Leda Miranda Hühne & $\begin{array}{l}\text { Heidegger's Philosophy - A New } \\
\text { Understanding of the World }\end{array}$ & \\
\hline 08/10/99 & $\begin{array}{l}\text { Thematic } \\
\text { presentation }\end{array}$ & Leandro Konder & The crisis of ethical values & \\
\hline 15/10/99 & $\begin{array}{l}\text { Thematic } \\
\text { presentation }\end{array}$ & $\begin{array}{l}\text { Sallete Ferreira, Marcelo } \\
\text { Santos Cruz }\end{array}$ & alcoholism and work environment & \\
\hline 22/10/99 & $\begin{array}{l}\text { Thematic } \\
\text { presentation }\end{array}$ & $\begin{array}{l}\text { Carlos Edson Duarte, Cloyra } \\
\text { de Paiva Almeida, Fernanda } \\
\text { Souza, Elie Cheniaux Júnior, } \\
\text { Isabela MMM Vieira }\end{array}$ & & \\
\hline 05/11/99 & $\begin{array}{l}\text { Thematic } \\
\text { presentation }\end{array}$ & $\begin{array}{l}\text { Katia de almeida martins, } \\
\text { Palm Beach/USA }\end{array}$ & $\begin{array}{l}\text { Therapy technique with children victims } \\
\text { of sexual abuse an experience with a } \\
\text { therapist in the USA }\end{array}$ & \\
\hline 12/11/99 & $\begin{array}{l}\text { Thematic } \\
\text { presentation }\end{array}$ & Marleide Mota Gomes & $\begin{array}{l}\text { The interface between epilepsy and } \\
\text { psychiatry }\end{array}$ & \\
\hline 19/11/99 & Simposium & & & \\
\hline 26/11/99 & $\begin{array}{l}\text { PhD thesis } \\
\text { defense }\end{array}$ & Yasmin Andrade Almeida & $\begin{array}{l}\text { Psychodynamic aspects of panic } \\
\text { disorder: a comparative analysis with } \\
\text { major disorder }\end{array}$ & \\
\hline 03/12/99 & $\begin{array}{l}\text { Thematic } \\
\text { presentation }\end{array}$ & $\begin{array}{l}\text { João Ferreira da Silva Filho, } \\
\text { Annette Leibing }\end{array}$ & IPUB's services and its integration & \\
\hline 10/12/99 & $\begin{array}{l}\text { PhD thesis } \\
\text { defense }\end{array}$ & Mary Yale Rodrigues Neves & $\begin{array}{l}\text { Teaching work and mental health: the } \\
\text { pain and the delight of being } \\
\text { (becoming) a teacher }\end{array}$ & \\
\hline 17/12/99 & $\begin{array}{l}\text { Thematic } \\
\text { presentation }\end{array}$ & Hower Kushner & $\begin{array}{l}\text { Does the biological revolution in } \\
\text { psychiatry eliminate psychological and } \\
\text { social explanations of behavior? }\end{array}$ & \\
\hline 12/01/01 & $\begin{array}{l}\text { Thematic } \\
\text { presentation }\end{array}$ & Francisco lancio Bastos & AIDS and Drugs Epidemiology & \\
\hline 19/01/01 & $\begin{array}{l}\text { Thematic } \\
\text { presentation }\end{array}$ & Daniel Groissman & $\begin{array}{l}\text { Elderly care policies: care models and } \\
\text { alternatives to institutionalization }\end{array}$ & \\
\hline 26/01/01 & $\begin{array}{l}\text { Thematic } \\
\text { presentation }\end{array}$ & $\begin{array}{l}\text { Ana Simões, Marcelo } \\
\text { Santos Cruz }\end{array}$ & $\begin{array}{l}\text { Research projects and assistance to } \\
\text { drug users }\end{array}$ & \\
\hline 09/03/01 & $\begin{array}{l}\text { Thematic } \\
\text { presentation }\end{array}$ & Nelson Maculan & $\begin{array}{l}\text { Year oppening class } \\
\text { Science and technology: artificial neural } \\
\text { networks; }\end{array}$ & \\
\hline 16/03/01 & $\begin{array}{l}\text { Thematic } \\
\text { presentation }\end{array}$ & $\begin{array}{l}\text { Pilar Belmonte, Valeria } \\
\text { Legrande } \mathrm{M} \text { dos Reis }\end{array}$ & Home monitoring in mental health & \\
\hline 23/03/01 & $\begin{array}{l}\text { Thematic } \\
\text { presentation }\end{array}$ & Anna Carolina Lo Bianco & Psychoanalysis in university & \\
\hline $30 / 03 / 01$ & $\begin{array}{l}\text { Thematic } \\
\text { presentation }\end{array}$ & Walter Zin & How to write an academic paper & \\
\hline
\end{tabular}




\begin{tabular}{|c|c|c|c|c|}
\hline Date & Event type & Presented by & Journal/Font & Event place \\
\hline 06/04/01 & $\begin{array}{l}\text { Thematic } \\
\text { presentation }\end{array}$ & $\begin{array}{l}\text { Alexandre Valença, Carla } \\
\text { Marques and Isabella } \\
\text { Nascimento }\end{array}$ & Anxiety and Depression Program & \\
\hline 20/04/01 & $\begin{array}{l}\text { Thematic } \\
\text { presentation }\end{array}$ & Carlos Mario Alvarez & Aging in Ferenczi & \\
\hline 27/04/01 & $\begin{array}{l}\text { Institutional } \\
\text { Congress }\end{array}$ & $\begin{array}{l}\text { Ana Cristina Figueiredo, } \\
\text { Sonia Sodre, Carmen } \\
\text { Falcão }\end{array}$ & $\begin{array}{l}\text { XXIV Institutional Congress of } \\
\text { specialization courses }\end{array}$ & \\
\hline 04/05/01 & $\begin{array}{l}\text { Thematic } \\
\text { presentation }\end{array}$ & $\begin{array}{l}\text { Carlos Edson Duarte, Cloira } \\
\text { Almeida, Elie Cheniaux }\end{array}$ & $\begin{array}{l}\text { The empirical method in psychoanalytic } \\
\text { investigation }\end{array}$ & \\
\hline $11 / 05 / 01$ & $\begin{array}{l}\text { Thematic } \\
\text { presentation }\end{array}$ & Edson Saggese & Adolescence and Psychosis & \\
\hline 19/05/01 & $\begin{array}{l}\text { Thematic } \\
\text { presentation }\end{array}$ & $\begin{array}{l}\text { Daniel Sampaio, from } \\
\text { Lisbon University }\end{array}$ & $\begin{array}{l}\text { Some considerations about family } \\
\text { psychotherapy }\end{array}$ & \\
\hline 25/05/01 & $\begin{array}{l}\text { Thematic } \\
\text { presentation }\end{array}$ & $\begin{array}{l}\text { Bianca Telles Ribeiro, Maci } \\
\text { Doria, Maria Tavares } \\
\text { Cavalcanti, Diana Pinto }\end{array}$ & Narrative, identity and clinics & \\
\hline 01/06/01 & $\begin{array}{l}\text { Thematic } \\
\text { presentation }\end{array}$ & $\begin{array}{l}\text { Mia Sandberg, from } \\
\text { Stockholm University }\end{array}$ & $\begin{array}{l}\text { Art therapy: the symbolic structural } \\
\text { method of art analysis }\end{array}$ & \\
\hline 08/06/01 & $\begin{array}{l}\text { Thematic } \\
\text { presentation }\end{array}$ & Jeremias Ferraz & $\begin{array}{l}\text { Displacement of the production axis of } \\
\text { psychoanalysis to Latin America }\end{array}$ & \\
\hline 11/06/01 & $\begin{array}{l}\text { Thematic } \\
\text { presentation }\end{array}$ & $\begin{array}{l}\text { Phillipa Hay, from Adelaide } \\
\text { University }\end{array}$ & $\begin{array}{l}\text { Eating disorders in Australia: } \\
\text { epidemiological and therapeutic aspects }\end{array}$ & \\
\hline 13/06/01 & $\begin{array}{l}\text { Thematic } \\
\text { presentation }\end{array}$ & Dr G Petrides & $\begin{array}{l}\text { Electroconvulsive therapy: new } \\
\text { perspectives }\end{array}$ & \\
\hline 22/06/01 & $\begin{array}{l}\text { Thematic } \\
\text { presentation }\end{array}$ & Jerson Laks & $\begin{array}{l}\text { Ability to drive cars in the elderly: an } \\
\text { assessment }\end{array}$ & \\
\hline 29/06/01 & $\begin{array}{l}\text { Thematic } \\
\text { presentation }\end{array}$ & $\begin{array}{l}\text { Marcelo Santos Cruz, } \\
\text { Salette Maria Barros } \\
\text { Ferreira }\end{array}$ & $\begin{array}{l}\text { Study and Assistance Program for Drug } \\
\text { Dependents IPUB/UFRJ }\end{array}$ & \\
\hline 06/07/01 & Homage & $\begin{array}{l}\text { Prof Eustachio Portella } \\
\text { Nunes Filho, Valentin Gentil } \\
\text { Filho }\end{array}$ & $\begin{array}{l}\text { Homage to the memmory of Prof José } \\
\text { Leme Lopes }\end{array}$ & \\
\hline 13/07/01 & $\begin{array}{l}\text { Thematic } \\
\text { presentation }\end{array}$ & $\begin{array}{l}\text { Madalena Pizzaia, Abmael } \\
\text { de Souza Alves, Maria das } \\
\text { Graças Ribeiro }\end{array}$ & $\begin{array}{l}\text { Luis Cerqueira daily care center: } \\
\text { psychosocial care device }\end{array}$ & \\
\hline 20/07/01 & $\begin{array}{l}\text { Thematic } \\
\text { presentation }\end{array}$ & $\begin{array}{l}\text { Miguel Chalub, Talvane } \\
\text { Moraes }\end{array}$ & Psychiatric reform in Brazil & \\
\hline 27/07/01 & $\begin{array}{l}\text { Thematic } \\
\text { presentation }\end{array}$ & $\begin{array}{l}\text { Cesar Augusto da Costa } \\
\text { Rodrigues, Katia Mecler }\end{array}$ & $\begin{array}{l}\text { Dangerousness: the unimpeachable } \\
\text { and the fully responsible }\end{array}$ & \\
\hline 03/08/01 & Homage & Ana Maria Pitta & $\begin{array}{l}\text { The importance of IPUB in mental } \\
\text { health care - IPUB anniversary } \\
\text { celebration }\end{array}$ & \\
\hline 09/08/01 & $\begin{array}{l}\text { Thematic } \\
\text { presentation }\end{array}$ & Cristina Redko & $\begin{array}{l}\text { Fighting against "evil": religious and } \\
\text { cultural constructions of the first } \\
\text { psychotic experience of young people } \\
\text { living in São Paulo }\end{array}$ & \\
\hline 10/08/01 & $\begin{array}{l}\text { Thematic } \\
\text { presentation }\end{array}$ & Michael L. Commons & $\begin{array}{l}\text { A new perspective on the psychological } \\
\text { boundaries between therapist and } \\
\text { patient }\end{array}$ & \\
\hline 17/08/01 & $\begin{array}{l}\text { Thematic } \\
\text { presentation }\end{array}$ & Mirian de Carvalho & $\begin{array}{l}\text { Psychiatric institution and architectural } \\
\text { space }\end{array}$ & \\
\hline 24/08/01 & $\begin{array}{l}\text { Thematic } \\
\text { presentation }\end{array}$ & Ligia Costa Leite & $\begin{array}{l}\text { Itinerant people from Brazil and Canada: } \\
\text { a matter of mental health }\end{array}$ & \\
\hline $31 / 08 / 01$ & $\begin{array}{l}\text { Thematic } \\
\text { presentation }\end{array}$ & $\begin{array}{l}\text { Roberto Airthon Piedade, } \\
\text { Adriana Fiszman }\end{array}$ & $\begin{array}{l}\text { New perspectives on quantitative } \\
\text { electroencephalography in psychiatry }\end{array}$ & \\
\hline
\end{tabular}




\begin{tabular}{|c|c|c|c|c|}
\hline Date & Event type & Presented by & Journal/Font & Event place \\
\hline $14 / 09 / 01$ & $\begin{array}{l}\text { Thematic } \\
\text { presentation }\end{array}$ & Elie Cheniaux Junior & $\begin{array}{l}\text { Premenstrual Syndrome: a meeting } \\
\text { point between psychiatry and } \\
\text { psychology }\end{array}$ & \\
\hline 21/09/01 & $\begin{array}{l}\text { Thematic } \\
\text { presentation }\end{array}$ & Fabiana Leão Lopes & Sleep Panic Attack: Preliminary Data & \\
\hline 28/09/01 & $\begin{array}{l}\text { Thematic } \\
\text { presentation }\end{array}$ & $\begin{array}{l}\text { Carla Meis, Salette Maria } \\
\text { Barros Ferreira }\end{array}$ & & \\
\hline 05/10/01 & $\begin{array}{l}\text { Thematic } \\
\text { presentation }\end{array}$ & Pedro Gabriel G Delgado & Mental health research and assistance & \\
\hline $19 / 10 / 01$ & & & Rio de Janeiro State Psychiatry Day & \\
\hline 26/10/01 & $\begin{array}{l}\text { Thematic } \\
\text { presentation }\end{array}$ & $\begin{array}{l}\text { Amanda Athayde, Leonardo } \\
\text { Fontenelle }\end{array}$ & $\begin{array}{l}\text { Gender identity disorder: } \\
\text { endocrinological and psychiatric } \\
\text { aspects }\end{array}$ & \\
\hline 09/11/01 & $\begin{array}{l}\text { Thematic } \\
\text { presentation }\end{array}$ & Ivan Figueira & PTSD reactions & \\
\hline $23 / 11 / 01$ & $\begin{array}{l}\text { Thematic } \\
\text { presentation }\end{array}$ & Diana Antonáz & $\begin{array}{l}\text { Pain and the meaning of life, a case } \\
\text { study: the "new disease" of telephone } \\
\text { operators in Rio de Janeiro } \\
(1980 / 1990)\end{array}$ & \\
\hline $30 / 11 / 01$ & $\begin{array}{l}\text { Thematic } \\
\text { presentation }\end{array}$ & $\begin{array}{l}\text { Ana Cristina Figueiredo, } \\
\text { Teresa Pinheiro, Sonia } \\
\text { Alberti }\end{array}$ & $\begin{array}{l}\text { The psychoanalytic method: research } \\
\text { and clinic }\end{array}$ & \\
\hline 07/12/01 & & $\begin{array}{l}\text { Vania Pires, John Kennedy, } \\
\text { Domingos Brnardo, Fausto } \\
\text { L. Gomes júnior, Marcelo } \\
\text { Santos Cruz }\end{array}$ & $\begin{array}{l}\text { I seminar adolescence, risk behavior } \\
\text { and drugs: preventive measures and } \\
\text { therapeutic actions }\end{array}$ & \\
\hline $14 / 12 / 01$ & $\begin{array}{l}\text { Thematic } \\
\text { presentation }\end{array}$ & Debora Miriam Raab Glina & Mental health at work & \\
\hline 21/12/01 & $\begin{array}{l}\text { Thematic } \\
\text { presentation }\end{array}$ & Maria Alice Bastos Silva & $\begin{array}{l}\text { Work and income generation in the area } \\
\text { of mental health }\end{array}$ & \\
\hline 09/03/17 & $\begin{array}{l}\text { Thematic } \\
\text { presentation }\end{array}$ & $\begin{array}{l}\text { Maria Tavares and Benilton } \\
\text { Bezerra }\end{array}$ & $\begin{array}{l}\text { Neurosciences, psychiatry and } \\
\text { psychoanalysis }\end{array}$ & \\
\hline $16 / 03 / 17$ & & $\begin{array}{l}\text { Octavio Domond and } \\
\text { Nelson Goldenstein }\end{array}$ & $\begin{array}{l}\text { Symposium of Psychopathology and } \\
\text { Subjectivity Laboratory (LEPPS) }\end{array}$ & \\
\hline 23/03/17 & & Paula Cerqueira & $\begin{array}{l}\text { Symposium of Multiprofessional } \\
\text { registrars }\end{array}$ & \\
\hline 06/04/17 & & Ana Cristina Figueiredo & $\begin{array}{l}\text { Symposium for Clinical Research in } \\
\text { Psychoanalysis (CLIPSA) }\end{array}$ & \\
\hline $13 / 04 / 17$ & & Marcelo Cruz & PROJAD Symposium & \\
\hline $20 / 04 / 17$ & & $\begin{array}{l}\text { Valeska Marinho, Andrea } \\
\text { Deslandes and Marcia } \\
\text { Dourado }\end{array}$ & $\begin{array}{l}\text { CDA symposium: Prevention and } \\
\text { treatment of mental illness in the elderly }\end{array}$ & \\
\hline 27/04/17 & & $\begin{array}{l}\text { Pedro Gabriel and Cristina } \\
\text { Ventura }\end{array}$ & $\begin{array}{l}\text { NUPPSAM Symposium ('Collaborative } \\
\text { Care in Psychosocial Care for Children } \\
\text { and Adolescents) }\end{array}$ & \\
\hline $04 / 05 / 17$ & & William Berger & PROPSAM Symposium & \\
\hline $11 / 05 / 17$ & & Emiliane Cunha Ferreira & Nurse week Symposium & \\
\hline $18 / 05 / 17$ & Homage & Abmael Alves & $\begin{array}{l}\text { Commemoration of the anti-asylum } \\
\text { campaing }\end{array}$ & \\
\hline $25 / 05 / 17$ & & Ana Cristina Figueiredo & $\begin{array}{l}\text { Professional Master's Program } \\
\text { Symposium }\end{array}$ & \\
\hline 08/06/17 & & Maria Tavares & $\begin{array}{l}40 \text { Alternative Mental Health Practices } \\
\text { Forum }\end{array}$ & \\
\hline $15 / 06 / 17$ & & Nathália Sabbagh & CARIM Symposium & \\
\hline $22 / 06 / 17$ & & Julio Verztman & NEPECC Symposium & \\
\hline
\end{tabular}




\begin{tabular}{|c|c|c|c|c|}
\hline Date & Event type & Presented by & Journal/Font & Event place \\
\hline 29/06/17 & & & IPUB's 80 years seminar & \\
\hline 06/07/17 & $\begin{array}{l}\text { Thematic } \\
\text { presentation }\end{array}$ & Jose Alberto Zuzman & $\begin{array}{l}\text { Dependence, Addiction and } \\
\text { Psychoanalysis }\end{array}$ & \\
\hline 03/08/17 & Homage & & IPUB's 80 years anniversarie & \\
\hline $10 / 08 / 17$ & & Antonio Egidio Nardi & $\begin{array}{l}\text { Panic and Breathing Laboratory } \\
\text { Symposium }\end{array}$ & \\
\hline $17 / 08 / 17$ & & Leonardo Fontenelle & $\begin{array}{l}\text { Symposium on Anxiety, Obsessions and } \\
\text { Compulsions Program }\end{array}$ & \\
\hline $24 / 08 / 17$ & & Elie Cheniaux & $\begin{array}{l}\text { Bipolar Disorder Research Laboratory } \\
\text { Symposium }\end{array}$ & \\
\hline $31 / 08 / 17$ & & Leonardo Meyer & Forensic Psychiatry Symposium & \\
\hline $14 / 09 / 17$ & & Ligia Costa Leite & $\begin{array}{l}\text { Adolescence and Mental Health } \\
\text { Symposium }\end{array}$ & \\
\hline 21/09/17 & & Ivan Figueira & $\begin{array}{l}\text { Post Traumatic Stress Disorder } \\
\text { Symposium }\end{array}$ & \\
\hline 28/09/17 & & $\begin{array}{l}\text { Antonio Egidio Nardi/Jose } \\
\text { Appolinario }\end{array}$ & $\begin{array}{l}\text { Resistant Depression Symposium } \\
\text { (DeReTrat) }\end{array}$ & \\
\hline 05/10/17 & $\begin{array}{l}\text { Thematic } \\
\text { presentation }\end{array}$ & Nuria Nalajovich & University Student Mental Health & \\
\hline $26 / 10 / 17$ & & Paulo Mattos & $\begin{array}{l}\text { Attention Deficit Hyperactivity Disorder } \\
\text { Symposium (NEDDA) }\end{array}$ & \\
\hline 09/11/17 & & José Carlos Appolinario & $\begin{array}{l}\text { Symposium on Obesity and Eating } \\
\text { Disorders (GOTA) }\end{array}$ & \\
\hline 23/11/17 & & Edson & Suicide Symposium & \\
\hline $30 / 11 / 17$ & & $\begin{array}{l}\text { Marcele Carvalho, Paula } \\
\text { Ventura and Rafael Freire }\end{array}$ & $\begin{array}{l}\text { Virtual Reality Symposium on the } \\
\text { Assessment and Treatment of Mental } \\
\text { Disorders }\end{array}$ & \\
\hline $07 / 12 / 17$ & & Heloisa Brasil & Child Psychiatry Symposium (SPIA) & \\
\hline 15/03/19 & $\begin{array}{l}\text { Thematic } \\
\text { presentation }\end{array}$ & Jorge Adelino & $\begin{array}{l}\text { Opening of the year - Medico-Legal } \\
\text { Documents }\end{array}$ & \\
\hline 22/03/19 & & $\begin{array}{l}\text { Dipex Internacional } \\
\text { Researchers (EUA, Austrália } \\
\text { and Canadá) }\end{array}$ & $\begin{array}{l}\text { International Seminar on narratives in } \\
\text { Health }\end{array}$ & \\
\hline 29/03/19 & $\begin{array}{l}\text { Thematic } \\
\text { presentation }\end{array}$ & Roberto Lent & $\begin{array}{l}\text { (Neuro)Science for Education: a bridge } \\
\text { between two worlds }\end{array}$ & \\
\hline 05/04/19 & $\begin{array}{l}\text { Thematic } \\
\text { presentation }\end{array}$ & Marco Antonio Brasil & $\begin{array}{l}\text { The psychiatric ward in the general } \\
\text { hospital: challenges and proposals }\end{array}$ & \\
\hline $12 / 04 / 19$ & $\begin{array}{l}\text { Thematic } \\
\text { presentation }\end{array}$ & Andrea Deslandes & Physical Exercise and Mental Health & \\
\hline 26/04/19 & $\begin{array}{l}\text { Thematic } \\
\text { presentation }\end{array}$ & Marcio Amaral & Four Affirmations to a Potential Suicide & \\
\hline 03/05/19 & $\begin{array}{l}\text { Thematic } \\
\text { presentation }\end{array}$ & Talvane Marins de Moraes & Psychiatric and psychological autopsy & \\
\hline 10/05/19 & $\begin{array}{l}\text { Thematic } \\
\text { presentation }\end{array}$ & Jerson Laks & $\begin{array}{l}\text { National Plan for dementia_what and } \\
\text { what it is for }\end{array}$ & \\
\hline $17 / 05 / 19$ & & $\begin{array}{l}\text { Jornada de Psiquiatria } \\
\text { Forense do IPUB }\end{array}$ & IPUB Forensic Psychiatry Conference & \\
\hline 24/05/19 & $\begin{array}{l}\text { Thematic } \\
\text { presentation }\end{array}$ & João Romildo Bueno & Psychopharmacology in dual disorders & \\
\hline $31 / 05 / 19$ & $\begin{array}{l}\text { Thematic } \\
\text { presentation }\end{array}$ & Paulo Mattos & ADHD: The Emperor of Misdiagnosis & \\
\hline 07/06/19 & $\begin{array}{l}\text { Thematic } \\
\text { presentation }\end{array}$ & Vanessa Rocha Rego & $\begin{array}{l}\text { Clinic and Neuroimaging of Post- } \\
\text { Traumatic Stress Disorder }\end{array}$ & \\
\hline
\end{tabular}




\begin{tabular}{|c|c|c|c|c|}
\hline Date & Event type & Presented by & Journal/Font & Event place \\
\hline $14 / 06 / 19$ & $\begin{array}{l}\text { Thematic } \\
\text { presentation }\end{array}$ & Antonio Egidio Nardi & $\begin{array}{l}\text { Panic Disorder: Perspectives in Medical } \\
\text { and Psychotherapeutic Diagnosis and } \\
\text { Treatment }\end{array}$ & \\
\hline 28/06/19 & & Maria Tavares & $\begin{array}{l}\text { V Alternative Mental Health Practices } \\
\text { Forum }\end{array}$ & \\
\hline 05/07/19 & $\begin{array}{l}\text { Thematic } \\
\text { presentation }\end{array}$ & Carolina Costa & $\begin{array}{l}\text { Smoke-Free Psychiatric Hospital: and } \\
\text { Possible? }\end{array}$ & \\
\hline 02/08/19 & $\begin{array}{l}\text { Thematic } \\
\text { presentation }\end{array}$ & Marcia Dourado & Social Cognition in Alzheimer's Disease & \\
\hline 09/08/19 & $\begin{array}{l}\text { Thematic } \\
\text { presentation }\end{array}$ & Alexandre Valença & Drugs and Law: Forensic Aspects & \\
\hline $16 / 08 / 19$ & $\begin{array}{l}\text { Thematic } \\
\text { presentation }\end{array}$ & Sergio Ferreira & $\begin{array}{l}\text { Connecting the Brain and the Body in } \\
\text { Dementia: A New Myokine Protects the } \\
\text { Brain in Preclinical Models of } \\
\text { Alzheimer's Disease }\end{array}$ & \\
\hline 23/08/19 & $\begin{array}{l}\text { Thematic } \\
\text { presentation }\end{array}$ & Ana Crisitna Figueiredo & $\begin{array}{l}\text { Clinical Research Center in } \\
\text { Psychoanalysis: the collective } \\
\text { construction of the Clinical case }\end{array}$ & \\
\hline $30 / 08 / 19$ & $\begin{array}{l}\text { Thematic } \\
\text { presentation }\end{array}$ & Rogerio Panizutti & $\begin{array}{l}\text { Use of digital technologies to promote } \\
\text { mental health }\end{array}$ & \\
\hline $13 / 09 / 19$ & $\begin{array}{l}\text { Thematic } \\
\text { presentation }\end{array}$ & Pedro Gabriel & $\begin{array}{l}\text { Violence and psychosocial care: } \\
\text { challenges for services in the territory }\end{array}$ & \\
\hline 20/09/19 & $\begin{array}{l}\text { Thematic } \\
\text { presentation }\end{array}$ & Debora Foguel & $\begin{array}{l}\text { A reflection on postgraduate studies } \\
\text { and research in Brazil }\end{array}$ & \\
\hline 27/09/19 & $\begin{array}{l}\text { Thematic } \\
\text { presentation }\end{array}$ & Leonardo Meyer & $\begin{array}{l}\text { Assessment of working capacity in } \\
\text { psychiatry: current perspectives }\end{array}$ & \\
\hline $18 / 10 / 19$ & $\begin{array}{l}\text { Thematic } \\
\text { presentation }\end{array}$ & Maria Tavares & Perspectives of mental health in Brazil & \\
\hline $25 / 10 / 19$ & & Julia Pimenta & Medicinal Cannabis Symposium & \\
\hline $01 / 11 / 19$ & $\begin{array}{l}\text { Thematic } \\
\text { presentation }\end{array}$ & William Berger & $\begin{array}{l}\text { The profound changes in the evaluation } \\
\text { criteria of Postgraduate courses by } \\
\text { CAPES and their impacts on PROPSAM }\end{array}$ & \\
\hline 08/11/19 & $\begin{array}{l}\text { Thematic } \\
\text { presentation }\end{array}$ & Gabriela Menezes & $\begin{array}{l}\text { Anxiety Disorders: The Challenges of } \\
\text { the } 21 \text { st Century }\end{array}$ & \\
\hline $22 / 11 / 19$ & & Beatriz Salles & Music Therapy Symposium & \\
\hline 29/11/19 & $\begin{array}{l}\text { Thematic } \\
\text { presentation }\end{array}$ & Valeska Marinho & The psychiatric clinic for the elderly & \\
\hline $06 / 12 / 19$ & & Pedro Gabriel & CAPS Symposium & \\
\hline $13 / 03 / 20$ & $\begin{array}{l}\text { Thematic } \\
\text { presentation }\end{array}$ & Jorge Adelino & Opening of the Study Center & \\
\hline $20 / 03 / 20$ & $\begin{array}{l}\text { Thematic } \\
\text { presentation }\end{array}$ & Antonio Egidio Nardi & Suicide & \\
\hline $27 / 03 / 20$ & $\begin{array}{l}\text { Thematic } \\
\text { presentation }\end{array}$ & Alexandre Valença & Anxiety disorders & \\
\hline 03/04/20 & $\begin{array}{l}\text { Thematic } \\
\text { presentation }\end{array}$ & Valeska Marinho & $\begin{array}{l}\text { Neuropsychiatric Symptoms in } \\
\text { Dementia }\end{array}$ & \\
\hline $17 / 04 / 20$ & $\begin{array}{l}\text { Thematic } \\
\text { presentation }\end{array}$ & Mariana Luz & PTSD and COVID-19 & \\
\hline $24 / 04 / 20$ & $\begin{array}{l}\text { Thematic } \\
\text { presentation }\end{array}$ & Maria Tavares & Mental Health in Times of Pandemic & \\
\hline 08/05/20 & $\begin{array}{l}\text { Thematic } \\
\text { presentation }\end{array}$ & Appolinario & $\begin{array}{l}\text { IMPACT OF COVID-19 ON EATING } \\
\text { BEHAVIOR }\end{array}$ & \\
\hline
\end{tabular}




\begin{tabular}{|c|c|c|c|c|}
\hline Date & Event type & Presented by & Journal/Font & Event place \\
\hline 22/05/20 & $\begin{array}{l}\text { Thematic } \\
\text { presentation }\end{array}$ & Andrea Deslandes & $\begin{array}{l}\text { Physical activity and mental health in } \\
\text { times of pandemic }\end{array}$ & \\
\hline 29/05/20 & $\begin{array}{l}\text { Thematic } \\
\text { presentation }\end{array}$ & Carolina Costa & $\begin{array}{l}\text { Smoking and Mental Health in Times of } \\
\text { Pandemic }\end{array}$ & \\
\hline 05/06/20 & $\begin{array}{l}\text { Thematic } \\
\text { presentation }\end{array}$ & Antonio Egidio Nardi & Anxiety and COVID-19 & \\
\hline $19 / 06 / 20$ & $\begin{array}{l}\text { Thematic } \\
\text { presentation }\end{array}$ & Juliana Pimenta & $\begin{array}{l}\text { Mental health of children and } \\
\text { adolescents in times of COVID-19 }\end{array}$ & \\
\hline 26/06/20 & $\begin{array}{l}\text { Thematic } \\
\text { presentation }\end{array}$ & Lisieux Borba Telles & Domestic Violence in Times of Covid-19 & \\
\hline 03/07/20 & $\begin{array}{l}\text { Thematic } \\
\text { presentation }\end{array}$ & Marcia Dourado & Dementia care in times of COVID-19 & \\
\hline 07/08/20 & $\begin{array}{l}\text { Thematic } \\
\text { presentation }\end{array}$ & Elie Cheniaux & $\begin{array}{l}\text { Bentinho's delusion of jealousy, in 'Dom } \\
\text { Casmurro', by Machado de Assis }\end{array}$ & \\
\hline $14 / 08 / 20$ & $\begin{array}{l}\text { Thematic } \\
\text { presentation }\end{array}$ & Rafael Thomaz & $\begin{array}{l}\text { How to deal with worries in times of } \\
\text { COVID-19 }\end{array}$ & \\
\hline 21/08/20 & $\begin{array}{l}\text { Thematic } \\
\text { presentation }\end{array}$ & Paulo Mattos & $\begin{array}{l}\text { Dementia Risk Factors and the context } \\
\text { of the COVID-19 pandemic }\end{array}$ & \\
\hline 28/08/20 & $\begin{array}{l}\text { Thematic } \\
\text { presentation }\end{array}$ & $\begin{array}{l}\text { Telemedicine/Luiz Ary } \\
\text { Messina }\end{array}$ & $\begin{array}{l}\text { Build, Collaborate and Disseminate } \\
\text { Knowledge Network }\end{array}$ & \\
\hline 04/09/20 & $\begin{array}{l}\text { Thematic } \\
\text { presentation }\end{array}$ & Ana Cristina Figueiredo & $\begin{array}{l}\text { The Singular and the Collective from the } \\
\text { perspective of Psychoanalysis: for a } \\
\text { policy of the 'not all' }\end{array}$ & \\
\hline $11 / 09 / 20$ & $\begin{array}{l}\text { Thematic } \\
\text { presentation }\end{array}$ & $\begin{array}{l}\text { Lia Rejane Mendes } \\
\text { Barcellos }\end{array}$ & $\begin{array}{l}\text { THE PATIENT AS NARRATOR OF HIS } \\
\text { HISTORY IN MUSIC THERAPY OR: THE } \\
\text { CLOSET GIRL }\end{array}$ & \\
\hline $18 / 09 / 20$ & $\begin{array}{l}\text { Thematic } \\
\text { presentation }\end{array}$ & Anna Lucia King & $\begin{array}{l}\text { Delete-Detox Digital Laboratory and } \\
\text { Conscious Use of Technologies/IPUB/ } \\
\text { UFRJ. Work carried out before and } \\
\text { during the Covid-19 pandemic. }\end{array}$ & \\
\hline 25/09/20 & $\begin{array}{l}\text { Thematic } \\
\text { presentation }\end{array}$ & Alexandre Valença & $\begin{array}{l}\text { Neuropsychiatric Disorders and } \\
\text { COVID-19 }\end{array}$ & \\
\hline 02/10/20 & $\begin{array}{l}\text { Thematic } \\
\text { presentation }\end{array}$ & José Carlos Appolinario & $\begin{array}{l}\text { FOOD COMPULSION IN RIO DE } \\
\text { JANEIRO: A MATTER OF WEIGHT? }\end{array}$ & \\
\hline 09/10/20 & $\begin{array}{l}\text { Thematic } \\
\text { presentation }\end{array}$ & Helena Moraes & $\begin{array}{l}\text { Integrative Practices and Mental Health: } \\
\text { A View of Neuroscience }\end{array}$ & \\
\hline $16 / 10 / 20$ & $\begin{array}{l}\text { Thematic } \\
\text { presentation }\end{array}$ & Octavio Dummond & Psychic Suffering and Social Suffering & \\
\hline $30 / 10 / 20$ & $\begin{array}{l}\text { Thematic } \\
\text { presentation }\end{array}$ & Mauricio Tostes & $\begin{array}{l}\text { Doctor-Patient Relationship: some } \\
\text { reflections }\end{array}$ & \\
\hline 06/11/20 & $\begin{array}{l}\text { Thematic } \\
\text { presentation }\end{array}$ & Evento EaD_Egidio Nardi & PROPSAM Distance Learning event & \\
\hline $13 / 11 / 20$ & $\begin{array}{l}\text { Thematic } \\
\text { presentation }\end{array}$ & Daniel Mograbi & $\begin{array}{l}\text { Self-awareness in neurological and } \\
\text { psychiatric patients }\end{array}$ & \\
\hline $27 / 11 / 20$ & $\begin{array}{l}\text { Thematic } \\
\text { presentation }\end{array}$ & Leonardo Fontenelle & $\begin{array}{l}\text { TRANSDIAGNOSTIC ASPECTS OF } \\
\text { OBSESSIONS, COMPULSIONS and } \\
\text { RELATED PHENOMENA }\end{array}$ & \\
\hline $04 / 12 / 20$ & $\begin{array}{l}\text { Thematic } \\
\text { presentation }\end{array}$ & Pedro Gabriel Delgado & $\begin{array}{l}\text { Barriers to access to mental health } \\
\text { treatment during the COVID pandemic } 19\end{array}$ & \\
\hline
\end{tabular}

